\title{
Local $\mathcal{P}$ Violation Effects and Thermalization in QCD: Views from Quantum Field Theory and Holography.
}

\author{
Ariel R. Zhitnitsky \\ Department of Physics $\&$ Astronomy, University of British Columbia, Vancouver, B.C. V6T 1Z1, Canada
}

\begin{abstract}
We argue that the local violation of $\mathcal{P}$ and $\mathcal{C P}$ invariance in heavy ion collisions and the universal thermal aspects observed in high energy collisions are in fact two sides of the same coin, and both are related to quantum anomalies of QCD. We argue that the low energy relations representing the quantum anomalies of QCD are saturated by coherent low dimensional vacuum configurations as observed in Monte Carlo lattice studies. The thermal spectrum and approximate universality of the temperature with no dependence on energy of colliding particles in this framework is due to the fact that the emission results from the distortion of these low dimensional vacuum sheets rather than from the colliding particles themselves. The emergence of the long- range correlations of $\mathcal{P}$ odd domains (a feature which is apparently required for explanation of the asymmetry observed at RHIC and LHC) is also a result of the same distortion of the QCD vacuum configurations. We formulate the corresponding physics using the effective low energy effective Lagrangian. We also formulate the same physics in terms of the dual holographic picture when low-dimensional sheets of topological charge embedded in 4d space, as observed in Monte Carlo simulations, are identified with D2 branes. Finally, we argue that study of these long range correlations in heavy ion collisions could serve as a perfect test of a proposal that the observed dark energy in present epoch is a result of a tiny deviation of the QCD vacuum energy in expanding universe from its conventional value in Minkowski spacetime.
\end{abstract}




\section{INTRODUCTION. MOTIVATION}

Recently it has become clear that quantum anomalies (chiral and conformal) play very important role in the macroscopic dynamics of relativistic fluids. Much of this progress is motivated by very interesting ongoing experiments on local $\mathcal{P}$ and $\mathcal{C P}$ violation in QCD as studied at RHIC and ALICE at the LHC [1-4]. It is likely that the observed asymmetry is due to charge separation effect $[5,6]$ as a result of the chiral anomaly, see details below.

Another, naively unrelated, but well established property of QCD, apparently also related to the quantum anomaly, can be formulated as follows. Over the years, hadron production studies in a variety of high energy collision experiments have shown a remarkably universal feature, indicating a universal hadronization temperature $T_{H} \sim(150-200) \mathrm{MeV}$. From $e^{+} e^{-}$annihilation to $p p$ and $p \bar{p}$ interactions and further to collisions of heavy nuclei, with energies from a few $\mathrm{GeV}$ up to the $\mathrm{TeV}$ range, the production pattern always shows striking thermal aspects, connected to an apparently quite universal temperature around $T_{H} \sim(150-200) \mathrm{MeV}$ [7]. It has been recently argued that this feature may also be related to the quantum anomaly, the conformal anomaly [8]. The basic idea behind this bold proposal is the observation that the effective Lagrangian which exactly accounts for the effects of the conformal anomaly indicates a presence of the event horizon [8], which eventually leads to the universal thermal Hawking-Unruh radiation, see details below.

Few arguments that these two naively unrelated phenomena may in fact be originated from the same non-perturbative QCD dynamics were recently presented in [9]. However, the arguments of ref. [9] were based on an effective description in terms of an acceleration "a" of a Rindler observer, while microscopical picture in terms of specific gauge configurations responsible for the effect was completely obscured in formulation [9]. The goal of the present paper is (at least partially) to fill this gap, and develop some technical tools which might be appropriate to address those hard questions.

Before we develop a suitable technique to deal with both these two phenomena, we review each effect separately as it is commonly treated today. Our next step is to construct the low energy effective Lagrangian which accounts for the quantum anomalies. The quantum anomalies are normally represented in a form of specific properties of two point correlation functions. We opted, however to code this information in a form of low energy effective Lagrangian. It allows us to study the relevant non-perturbative gauge configurations which are responsible for saturation of the low energy theorems representing the quantum anomalies. The corresponding analysis will be supported by some recent lattice results as well as holographic interpretation of these lattice results.

\section{A. Local $\mathcal{P}$ and $\mathcal{C P}$ violation in QCD. Charge separation effect}

We start with review of the charge separation effect $[5,6]$ which can be explained in the following simple way. Let us assume that an effective $\theta(\vec{x}, t)_{\text {ind }} \neq 0$ is induced as a result of some non- equlibrium dynamics as suggested in refs. $[10-13]$. The $\theta(\vec{x}, t)_{\text {ind }}$ parameter enters the effective lagrangian as follows, $\mathcal{L}_{\theta}=-\theta_{\text {ind }} q$ where $q \equiv \frac{g^{2}}{64 \pi^{2}} \epsilon_{\mu \nu \rho \sigma} G^{a \mu \nu} G^{a \rho \sigma}$ such that local $\mathcal{P}$ and $\mathcal{C P}$ invariance of QCD is broken on the scales where correlated $\theta(\vec{x}, t)_{\text {ind }} \neq 0$ is induced. As a result of this violation, one should expect a number of $\mathcal{P}$ and $\mathcal{C P}$ violating effects taking place in the region where $\theta(\vec{x}, t)_{\text {ind }} \neq 0$.

This area of research became a very active field in recent years mainly due to very interesting ongoing experiments $[1-$ 4]. There is a number of different manifestations of this local $\mathcal{P}$ and $\mathcal{C P}$ violation, see [14-18] and many additional references therein. In particular, in the presence of an external magnetic field $\vec{B}$ or in case of the rotating system with angular velocity $\vec{\Omega}$ there will be induced electric current directed along $\vec{B}$ or $\vec{\Omega}$ correspondingly, resulting in separation of charges along those directions as mentioned above. One can interpret the same effects as a generation of induced electric field $\vec{E}$ directed along $\vec{B}$ or $\vec{\Omega}$ resulting in corresponding electric current flowing along $\vec{J} \sim \vec{B}$ or $\vec{J} \sim \vec{\Omega}$ directions. All these phenomena are obviously $\mathcal{P}$ and $\mathcal{C P}$ odd effects. Non-dissipating, induced vector current density has the form:

$$
\vec{J}=\left(\mu_{L}-\mu_{R}\right) \frac{e \vec{B}}{2 \pi^{2}},
$$

where $\mathcal{P}$ odd effect is explicitly present in this expression as the difference of chemical potentials of the right $\mu_{R}$ and left $\mu_{L}$ handed fermions is assumed to be nonzero, $\left(\mu_{L}-\mu_{R}\right) \neq 0$ in the region where $\theta(\vec{x}, t)_{\text {ind }} \neq 0$. The combination $\left(\mu_{L}-\mu_{R}\right)$ can be thought as $\dot{\theta}(t)$ after a corresponding $U(1)_{A}$ chiral time-dependent rotation is performed, see also [16] for a physical interpretation of the relation $\left(\mu_{L}-\mu_{R}\right)=\dot{\theta}(t)_{i n d}$. It is important to emphasize that the region where $\left\langle\theta(\vec{x}, t)_{\text {ind }}\right\rangle \neq 0$ should be much larger in size than the scale of conventional QCD fluctuations with correlation length $\sim \Lambda_{Q C D}^{-1}$

Closely related phenomena have been previously discussed in the physics of neutrinos [19] and quantum wires [20]. In QCD context formula (1) has been used in applications to neutron star physics where magnetic field is known to be 
large, and the corresponding $\left(\mu_{L}-\mu_{R}\right) \neq 0$ can be generated in neutron star environment as a result of continuos $\mathcal{P}$ violating processes happening in nuclear matter [21, 22]. It has been also applied to heavy ion collisions where an effective $\left(\mu_{L}-\mu_{R}\right) \neq 0$ is locally induced. The effect was estimated using the sphaleron transitions generating the topological charge density in the QCD plasma [14, 15]. The effect was coined as "chiral magnetic effect" (CME) [14, 15]. Formula (1) has been also derived a numerous number of times using varies techniques such as: effective lagrangian approach developed in [23]; explicit mode's summation [24]; direct lattice computations [17, 18]. In addition, the effect has been studied in holographic models of QCD [25, 26].

To summarize: on the theoretical side the effect is well established phenomenon. Future experiments at RHIC and LHC hopefully will teach us much more on implementation of this effect to heavy ion collisions, see review [27] with large number of references on recent developments. Furthermore, as all measurements are in fact $\mathcal{P}$ even observables, there are many background processes which considerably contribute to the effect [28-32], see some comments on this matter in section IV B. Therefore, it remains to be seen if the CME/charge separation effect is a main source of the observed event-by-event fluctuations. In this work we assume this is to be the case, and interpret the observations at RHIC and the LHC as a manifestation of the CME.

One of the crucial questions for the applications of the CME to heavy ion collisions is a correlation length of the induced $\left\langle\theta(\vec{x}, t)_{\text {ind }}\right\rangle \neq 0$ : why the $\mathcal{P}$ odd domains are large, much larger than conventional $\Lambda_{Q C D}^{-1}$ scale? Apparently, a relatively large correlation length is a required feature for interpretation of the observed asymmetry [1-4] in terms of CME as the conventional QCD vacuum processes are too small to explain the observed asymmetry [33]. This is in fact, the key element to be addressed in this paper: why the correlation length of $\mathcal{P}$ odd fluctuations could be large in heavy ion collisions?

\section{B. Universal hadronization temperature $T_{H} \sim(150-200) \mathrm{MeV}$}

The second part of the story goes as follows. While experimentally universal temperature around $T_{H} \sim(150-$ 200) $\mathrm{MeV}$ [7] is well established phenomenon, its origin is difficult to understand as number of incident particles in $e^{+} e^{-}$annihilation as well as in $p p$ and $p \bar{p}$ interactions is not sufficient even to talk about statistical averages. This observation motivated a number of early attempts [34] to interpret the resulting spectrum of particles as the HawkingUnruh radiation [35] when the event horizon emerges as a result of strong interactions with an effective temperature

$$
T=\frac{\mathbf{a}}{2 \pi} \sim \Lambda_{Q C D}
$$

being expressed in terms of effective deceleration parameter "a". Different aspects of this idea were advocated in a number of recent papers, see e.g. $[8,36]$. The problem of calculating of the effective parameter "a" is obviously very hard problem of strongly interacting QCD.

One should emphasize that the Planck spectrum in this approach is not resulted from the kinetics when the thermal equilibrium with temperature $T$ given by eq. (2) is reached due to the large number of collisions. Rather, the Planck spectrum in high energy collisions in this framework is resulted from the stochastic tunnelling processes when no information transfer occurs. In such circumstances the spectrum must be thermal. Such interpretation would naturally explain both puzzles of the phenomena:

a) the thermal spectrum in $e^{+} e^{-}, p p$ and $p \bar{p}$ high energy collisions emerges in spite of the fact that the statistical thermalization could never be reached in those systems;

b) an approximate universality of the temperature as it is expressed in terms of unique and fundamental scale $\Lambda_{Q C D}$ entering formula (2) with no dependence on energy of colliding particles in this formula.

The paper is organized as follows. Section II is devoted to the discussions of low energy relations in pure gluodynamics with no quarks. The corresponding relations can be conveniently represented in terms of the low energy Lagrangian for the background dilaton $\eta(x)$ and axion $a(x)$ fields combined into a single complex field which describes the dynamics of scalar $\eta \sim \frac{\alpha_{s}}{8 \pi} G_{\mu \nu} G^{\mu \nu}$ and pseudo scalar $a \sim \frac{\alpha_{s}}{8 \pi} G_{\mu \nu} \tilde{G}^{\mu \nu}$ components of the underlining gauge theory. This will allow us to study the long range effects of gauge configurations on scales which are much larger than conventional $\Lambda_{Q C D}^{-1}$ scale. The basic reason for emergence of this long range order is the $2 \pi$ periodic properties of the axion field $a(x)$ which is a crucial feature of QCD. This periodicity may result in formation of the topological solitons characterized by long range order. In section III we discuss some recent results from lattice simulations and holographic description as they seen from effective Lagrangian description. We shall identify the long range structure observed in lattice simulations with D2 branes in holographic description and with the axion field solitons in the effective Lagrangian approach. In section IV we discuss some applications of these ideas. Specifically, in section IV B we discuss local $\mathcal{P}$ violation effects and universality of hadronization temperature $T_{H}$ in high energy collisions, while in section IV C we discuss applications to cosmology. 


\section{EFFECTIVE LAGRANGIAN IN GLUODYAMICS}

In next subsection II A we review the low energy relations in gluodynamics. In subsection II B we construct the low energy effective lagrangian which is generating functional for these low energy correlation functions.

\section{A. Low energy relations}

In what follows we consider two types of low energy correlation functions (scalar and pseudoscalar) in gluodynamics. For the scalar channel case, it was shown long ago in ref. [37] that these correlation functions are fixed by renormalizability and conformal anomaly in gluodynamics,

$$
\lim _{q \rightarrow 0} i \int d x e^{i q x}\left\langle 0\left|T\left\{\frac{\beta\left(\alpha_{s}\right)}{4 \alpha_{s}} G^{2}(x) \frac{\beta\left(\alpha_{s}\right)}{4 \alpha_{s}} G^{2}(0)\right\}\right| 0\right\rangle=-4\left\langle\frac{\beta\left(\alpha_{s}\right)}{4 \alpha_{s}} G^{2}\right\rangle,
$$

where the one-loop $\beta$-function, $\beta\left(\alpha_{s}\right)=-b \alpha_{s}^{2} /(2 \pi)$ with $b=(11 / 3) N_{c}$ and $N_{c}$ stands for the number of colours. Arbitrary n-point functions of the trace of the energy-momentum tensor

$$
4 E_{\mathrm{v} a c} \equiv\langle\sigma\rangle=\left\langle\frac{\beta\left(\alpha_{s}\right)}{4 \alpha_{s}} G^{2}\right\rangle=\left\langle\frac{-b \alpha_{s}}{8 \pi} G^{2}\right\rangle+O\left(\alpha_{s}^{2}\right)
$$

can be obtained by further differentiating relation (3) :

$$
i^{n} \int d x_{1} \ldots d x_{n}\left\langle 0\left|T\left\{\sigma\left(x_{1}\right) \ldots \sigma\left(x_{n}\right) \sigma(0)\right\}\right| 0\right\rangle=(-4)^{n}\langle\sigma\rangle
$$

where, as in Eq.(3), a limiting procedure of the vanishing momentum transfer $q_{\mu}$ is implied.

Let us now address zero momentum correlation functions of the topological density operator in gluodynamics, the so-called topological susceptibility $\chi$. It can be written as

$$
\begin{aligned}
\chi & \equiv-\frac{\partial^{2} E_{\mathrm{v} a c}}{\partial \theta^{2}}=\lim _{q \rightarrow 0} i \int d x e^{i q x}\left\langle 0\left|T\left\{\frac{\alpha_{s}}{8 \pi} G \tilde{G}(x) \frac{\alpha_{s}}{8 \pi} G \tilde{G}(0)\right\}\right| 0\right\rangle \\
& =\xi^{2}\left\langle\frac{\beta\left(\alpha_{s}\right)}{4 \alpha_{s}} G^{2}\right\rangle=\frac{1}{N_{c}^{2}} E_{\mathrm{v} a c}(\theta),
\end{aligned}
$$

where $\xi$ stands for a generally unknown numerical coefficient (note that its $N_{c}$ dependence is expected to be $\xi \sim N_{c}^{-1}$, in order to match Witten-Veneziano [38-40] resolution of the $U_{A}(1)$ problem, see also [41-43]). It has been argued in $[44,45]$ that the corresponding consistent effective Lagrangian can be constructed for any rational $\xi=q / p$. However, in what follows, without loosing any generality, we consider the simplest and most appealing case when $\xi=\frac{1}{2 N_{c}}$ which corresponds to $E_{\mathrm{v} a c}(\theta) \sim \cos \left(\theta / N_{c}\right)$ behaviour for the vacuum energy. Such a behaviour is realized in all supersymmetric cases. The same $\cos \left(\theta / N_{c}\right)$ structure emerges even when supersymmetry is slightly broken, see details and references in [44, 45]. Furthermore, such a behaviour is also supported by the Veneziano construction [38] when the Veneziano ghost saturates all relevant $2 n$ - point correlation functions generalizing (6)

$$
\frac{\partial^{(2 n-1)}}{\partial \theta^{(2 n-1)}}\langle q(0)\rangle \sim \int d x_{1} \ldots d x_{2 n-1}\left\langle 0\left|T\left\{q\left(x_{1}\right) \ldots q\left(x_{2 n-1}\right) q(0)\right\}\right| 0\right\rangle \sim \frac{E_{\mathrm{v} a c}}{N_{c}^{2 n}} .
$$

Most importantly, the same behaviour can be demonstrated to emerge [46] in four dimensional "deformed QCD" formulated in [47] where all computations can be explicitly performed as the model is in a weak coupling regime. In the "deformed QCD" the behaviour (7) is direct manifestation of topological structure of the ground state when the non-trivial topological sectors of the theory and transitions between them are described in terms of the weakly coupled monopoles. The basic reason why eq. (7) holds is that all the correlation functions as well as the vacuum energy $E_{\mathrm{v} a c}$ are saturated by the same vacuum fluctuations which have non-dispersive nature, contribute to (6), (7) with "wrong sign", and can not be associated with any physical propagating degrees of freedom, see [46] for the technical details.

We note finally that the correlation functions $(6),(7)$ are defined via the path integral, i.e. with Wick's T-product in contrast with conventional Dyson's T-product when only physical asymptotic states contributes to the corresponding correlation functions, see Appendix of ref. [48] for discussions on some subtleties in the energy definition. 


\section{B. Effective Lagrangian}

The purpose of this section is to review the construction of the low energy effective Lagrangian for gluodynamics [44, 45]. The corresponding Effective Lagrangian contains all information provided by the low energy relations in the scalar (3) and pseudoscalar (6) channels including all multi-point correlation functions of operators $G^{2}$ and $G \tilde{G}$, which can be obtained by differentiating the two-point functions (3) and (6) with respect to $1 / g^{2}$ and $\theta$, see e.g. Eq.(5), (7).

Before proceeding with the presentation, we would like to pause for a comment on the meaning of this effective Lagrangian. As there exist no Goldstone bosons in pure gluodynamics, no Wilsonian effective Lagrangian, which would correspond to integrating out heavy modes, can be constructed for gluodynamics. Instead, one speaks in this case of an effective Lagrangian as a generating functional for vertex functions of the composite fields $G^{2}$ and $G \tilde{G}$. Moreover, only the potential part of this Lagrangian can be computed as it corresponds to zero momentum n-point functions of $G^{2}, G G$, fixed by the low energy theorems. Thus, such an effective Lagrangian is not very useful for calculating the S-matrix elements, but is perfectly suitable for addressing the vacuum properties of the theory, including the coordinate dependent vacuum configurations if they are treated as the background fields, see next section.

The task of constructing an effective Lagrangian can be considerably simplified as suggested in [44, 45] by going over to linear combinations of original operators which enter relations (3), (6) :

$$
H=\left(-\frac{b}{64 \pi^{2}} G^{2}+i \frac{N_{c}}{16 \pi^{2}} G \tilde{G}\right), \bar{H}=\left(-\frac{b}{64 \pi^{2}} G^{2}-i \frac{N_{c}}{16 \pi^{2}} G \tilde{G}\right) .
$$

In terms of these combinations, the low energy relations for renormalized zero momentum Green function, Eqs. (3) and (6), take particularly simple forms (for an arbitrary value of the vacuum angle $\theta$ ):

$$
\begin{aligned}
& \lim _{q \rightarrow 0} i \int d x e^{i q x}\langle 0|T\{H(x) H(0)\}| 0\rangle=-4\langle H\rangle, \\
& \lim _{q \rightarrow 0} i \int d x e^{i q x}\langle 0|T\{\bar{H}(x) \bar{H}(0)\}| 0\rangle=-4\langle\bar{H}\rangle, \\
& \lim _{q \rightarrow 0} i \int d x e^{i q x}\langle 0|T\{\bar{H}(x) H(0)\}| 0\rangle=0 .
\end{aligned}
$$

It is easy to check that the decoupling of the fields $H$ and $\bar{H}$ holds for arbitrary n-point functions of $H, \bar{H}$. This circumstance makes it particularly convenient to work with fields (8).

We now formulate the effective low energy Lagrangian reproducing at the tree level all low energy relations for the composite fields $H, \bar{H}$ discussed in previous subsection II A,

$$
\begin{aligned}
e^{-i V F(h, \bar{h})} & =\sum_{n=-\infty}^{+\infty} \exp \left\{-\frac{i V}{4}\left(h \ln \frac{h}{2 e\left|E_{\mathrm{v} a c}\right|}+\bar{h} \ln \frac{\bar{h}}{2 e\left|E_{\mathrm{v} a c}\right|}\right)\right. \\
& \left.+i \pi V\left(\frac{\theta+2 \pi n}{2 \pi N_{c}}\right) \frac{h-\bar{h}}{2 i}\right\}
\end{aligned}
$$

where $V$ is the 4 -volume of the system, and the effective zero momentum fields $h, \bar{h}$ satisfy the equations

$$
\int d x h=\left\langle\int d x H\right\rangle, \quad \int d x \bar{h}=\left\langle\int d x \bar{H}\right\rangle .
$$

For the case of single "dilaton" field $\sigma=-b \alpha_{s} /(8 \pi) G^{2}$, a similar problem of constructing an effective Lagrangian was solved long ago [49], see also some applications of these ideas in given context in refs [50-52]. To reproduce the dilaton potential constructed in [49] one should parametrize $h=\bar{h}=2\left|E_{\mathrm{v} a c}\right| \exp (\eta)$ in formula (10) to arrive to

$$
F(\eta)=-\left|E_{\mathrm{v} a c}\right| e^{\eta}(1-\eta), \quad 4 E_{\mathrm{v} a c} \equiv\langle\sigma\rangle=\left\langle\frac{-b \alpha_{s}}{8 \pi} G^{2}\right\rangle .
$$

The minimum of the potential is positioned at $\eta_{\min }=0$ which determines the ground state of the system to be $F_{\min }(\eta=0)=-\left|E_{\mathrm{v} a c}\right|$.

The generalization of this construction was suggested in [44, 45]. It is expressed by formula (10) and contains two new elements. First, it has an additional axion ${ }^{1}$ field " $a$ " along with the dilaton field $\eta$ defined as follows:

$$
h=2\left|E_{\mathrm{v} a c}\right| e^{\eta+i a}, \bar{h}=2\left|E_{\mathrm{v} a c}\right| e^{\eta-i a} .
$$

\footnotetext{
${ }^{1}$ We use term "axion" which is a jargon here. There is no real new dynamical degree of freedom such as axion, see recent reviews [53] about physical axion as real degree of freedom. However, the vacuum expectation value of $a$ field in $\theta$ vacua is directly related to $\theta$ as follows $\langle a\rangle=\theta / N_{c}$, see eq. (14) which justifies our terminology as the dynamical $\theta(x)$ is, by definition, the axion.
} 
This new " $a$ " field describes the dynamics of the pseudoscalar component $G \tilde{G}$ represented by low energy relations (6),(7). The second new element is that the structure of the effective potential $F(h, \bar{h})$ is such that it contains along with conventional "dynamical" part represented by the first term in the exponent in eq. (10) also the "topological" part represented by the second term in the exponent in eq. (10). The conventional "dynamical" part is merely a kinematical reformulation of the content of the low energy relations (9). The "topological" term on other hand does not modify the local relations (9), but rather, guarantees the $2 \pi$ periodicity $\theta \rightarrow \theta+2 \pi n$ of the energy of the ground state of the system. Indeed, the minimization of the effective potential (10) leads to the following result for the ground state $[44,45]$ :

$$
\begin{aligned}
F_{\min }(\theta) & =-\lim _{V \rightarrow \infty} \frac{1}{V} \ln \left\{\sum_{n=0}^{N_{c}-1} \exp \left[V\left|E_{\mathrm{v} a c}\right| \cos \left(\frac{\theta+2 \pi n}{N_{c}}\right)\right]\right\}, \\
\eta_{\text {min }} & =0, \quad a_{\min }=\frac{\theta+2 \pi l}{N_{c}} .
\end{aligned}
$$

The vacuum energy $F_{\min }(\theta)$ is obviously $2 \pi$ periodic function in spite of the fact that $\theta$ parameter enters the expression for the energy as $\theta / N_{c}$ in accordance with Witten-Veneziano resolution of the $U_{A}(1)$ problem represented by formula (6). This important property is achieved due to the "topological" part in effective potential (10). This term ensures that the effective potential has not one, but rather $N_{c}$ physically different local extrema. The physics is perfectly periodic ${ }^{2}$ in $\theta$ with period $2 \pi$, as the minima interchanging under the shift $\theta \rightarrow \theta+2 \pi$. At the same time, we observe level crossing with a two-fold degeneracy at $\theta=\pi(\bmod 2 \pi)$. The construction of the potential similar to $(12)$ when the axion field is taken into account leads to the following expression

$$
F(\eta(x), a(x))=-\left|E_{\mathrm{v} a c}\right|\left[e^{\eta}(1-\eta) \cos a+e^{\eta} a \sin a\right],
$$

where we keep only the lowest branch in expression (10) to simplify notations. This formula reduces, of course, to previous expression (12) in the limit $a \rightarrow 0$ when the axion field is neglected. One should remark here that the expression for $F(\eta, a)$ is not literally periodic $a \rightarrow a+2 \pi$ because $F(\eta(x), a(x))$ in eq. (15) corresponds to the lowest branch with $l=0$. However, the $2 \pi$ periodicity is restored when summation over all branches in partition function is implemented, similar to our procedure leading to eqs.(10), (14).

The corresponding discrete set of degenerate vacuum states as a result of the $2 \pi$ periodicity of the effective potential (10) for the axion field is a signal that the domain wall configurations interpolating between these states are present in the system. However, the corresponding configurations are not conventional domain walls similar to the well known ferromagnetic domain walls in condensed matter physics which interpolate between physically distinct vacuum states. In contrast, in present case a corresponding configuration interpolates between topologically different but physically equivalent winding states $|n\rangle$, which are connected to each other by large gauge transformation operator, see next section with elaboration on this issue. Axion field acts as a probe to signal that the degeneracy is present in the system and the presence of the domain walls is expected in the system. The corresponding domain wall configurations in Euclidean space will be interpreted in next section as configurations describing the tunnelling processes in Minkowski space, similar to Euclidean instantons. This interpretation should be contrasted with conventional interpretation of static domain walls defined in Minkowski space. Most important lesson from these discussions is as follows. The effective potential (10) which is generating functional for the low energy relations hints on the presence of the unusual topological configurations due to its generic $2 \pi$ periodicity. The physical meaning of these configurations will be elaborated later in the text.

\section{INSIGHTS FROM LATTICE SIMULATIONS AND FROM HOLOGRAPHIC PICTURE OF QCD}

In this section we want to get some insights on crucial vacuum configurations from the lattice results. The Monte Carlo simulations are normally performed in Euclidean space. Therefore, we reformulate the low energy relations discussed in previous section II to Euclidean space time in order to make comparison with lattice results.

\footnotetext{
2 Such a pattern is known to emerge in many four dimensional supersymmetric models, and also gluodynamics in the limit $N_{c}=\infty$. It has been further argued $[44,45]$ that the same pattern also emerges in four dimensional gluodynamics at any finite $N_{c}$. The same pattern emerges in holographic description of QCD [54] at $N_{c}=\infty$ as well. Finally, this pattern is realized in weakly coupled "deformed QCD" model where all computations are under complete theoretical control [46].
} 


\section{A. Topological susceptibility}

The scalar correlation function in Euclidean space takes the form and it is negative

$$
\int d x\left\langle 0\left|T\left\{\frac{b \alpha_{s}}{8 \pi} G^{2}(x) \frac{b \alpha_{s}}{8 \pi} G^{2}(0)\right\}\right| 0\right\rangle=-4\left\langle 0\left|\frac{b \alpha_{s}}{8 \pi} G^{2}\right| 0\right\rangle \quad<0,
$$

while the topological susceptibility in the Euclidean space is positive

$$
\chi_{E u c l}=\int d x\left\langle 0\left|T\left\{\frac{\alpha_{s}}{8 \pi} G \tilde{G}(x) \frac{\alpha_{s}}{8 \pi} G \tilde{G}(0)\right\}\right| 0\right\rangle=\frac{1}{N_{c}^{2}}\left|E_{\mathrm{v} a c}(\theta)\right|>0 .
$$

The difference in signs ${ }^{3}$ between these two correlation functions can be seen in Minkowski space as well, see eq. (3) versus (6). The crucial observation here is as follows: any physical state contributes to $\chi_{E u c l}$ with negative sign

$$
\chi_{\text {dispersive }} \sim \lim _{k \rightarrow 0} \sum_{n} \frac{\langle 0|q| n\rangle\langle n|q| 0\rangle}{-k^{2}-m_{n}^{2}}<0,
$$

in drastic contrast with low energy relation (17). It poses no problem for the correlation function (16) when the physical dilaton saturates the negative sign in eq.(16). At the same time the positive physical mass $m_{\eta^{\prime}}^{2}>0$ for the $\eta^{\prime}$ meson requires the positive sign for the topological susceptibility (17), see the original reference [39] for a thorough discussion. Therefore, there must be a contact contribution to $\chi$, which is not related to any propagating physical degrees of freedom, and it must have a "wrong sign" (in comparison with (18) representing the conventional dispersive contribution) to saturate the positive sign for topological susceptibility (17). In different words, it must be a non-dispersive contribution to $\chi$ which is not associated with any asymptotical physical states in conventional dispersion relations. In the framework [40] the contact term with "wrong sign" has been postulated, while in refs.[38, 39] the Veneziano ghost had been introduced to saturate the required property (17).

The simplest way to convince oneself in necessity for a non-dispersive contribution to $\chi$ with a "wrong sign" is to compute the topological susceptibility $\chi_{Q C D}$ in QCD rather than in gluodynamics. The topological susceptibility $\chi_{Q C D}\left(m_{q}=0\right)=0$ must vanish in the chiral limit as a consequence of the Ward Identities (WI). It is very instructive to see how it happens. If one models the contact contribution to $\chi$ using the Veneziano ghost, the topological susceptibility in Euclidean space can be represented as follows, see [9, 55] and references therein:

$$
\chi_{Q C D} \equiv \int d^{4} x\langle 0|T\{q(x), q(0)\}| 0\rangle_{Q C D}=\frac{f_{\eta^{\prime}}^{2} m_{\eta^{\prime}}^{2}}{4} \cdot \int d^{4} x\left[\delta^{4}(x)-m_{\eta^{\prime}}^{2} D^{c}\left(m_{\eta^{\prime}} x\right)\right]
$$

where $D^{c}\left(m_{\eta^{\prime}} x\right)$ is the Green's function of a free massive particle with standard normalization $\int d^{4} x m_{\eta^{\prime}}^{2} D^{c}\left(m_{\eta^{\prime}} x\right)=1$. The term proportional $-D^{c}\left(m_{\eta^{\prime}} x\right)$ with negative sign in eq. (19) is resulted from the lightest physical $\eta^{\prime}$ state of mass $m_{\eta^{\prime}}$ and it has a negative sign in accordance with (18). At the same time the $\delta^{4}(x)$ represents the ghost contribution with "wrong" sign which can not be associated with any physical states. The ghost's contribution can be also thought as the Witten's contact term [40] not related to any propagating degrees of freedom. The topological susceptibility $\chi_{Q C D}\left(m_{q}=0\right)=0$ vanishes in the chiral limit as a result of exact cancellation between two terms entering (19) in complete accordance with WI. The WI can not be satisfied if the contact term is not present in the system. When $m_{q} \neq 0$ the cancellation is not complete and $\chi_{Q C D} \simeq m_{q}\langle\bar{q} q\rangle$ in accordance with WI.

In case of "deformed QCD" considered in [46] we could explicitly compute the contact term and see that it is saturated by the monopoles which in weak coupling regime describe the tunnelling processes between different topological sectors of the theory. While the topological sectors in case of strongly coupled 4d QCD of course still exist, we do not have such luxury to compute the corresponding transitions explicitly. Nevertheless, we can still study the same dynamics of the topological sectors (and transitions between them) by using the ghost as an effective, but not physical, degree of freedom, which leads to expression (19). This formula being derived in strongly coupled QCD using the effective ghost description can be compared with the lattice results, see detail studies of this question in refs. [56-60]. In particular, one can explicitly see that the singular behaviour of the contact term is not an artifact of any approximation, but an inherent feature of underlying gauge theory. Furthermore, there are no any physical scale factors (such as $\Lambda_{\mathrm{QCD}}$ ) which would determine the singular behaviour of this term. In different words, the non-dispersive contribution in the lattice simulations has diverging nature of the core and vanishing width in the continuum limit, in full agreement with our eq. (19).

3 A warning signal with the signs: the physical degrees of freedom in Euclidean space (where the lattice computations are performed) contribute to topological susceptibility $\chi_{Q C D}$ with the negative sign, while the contact term (the Veneziano ghost) contributes with the positive sign, in contrast with our discussions in Minkowski space, see eqs. (3), (6). 


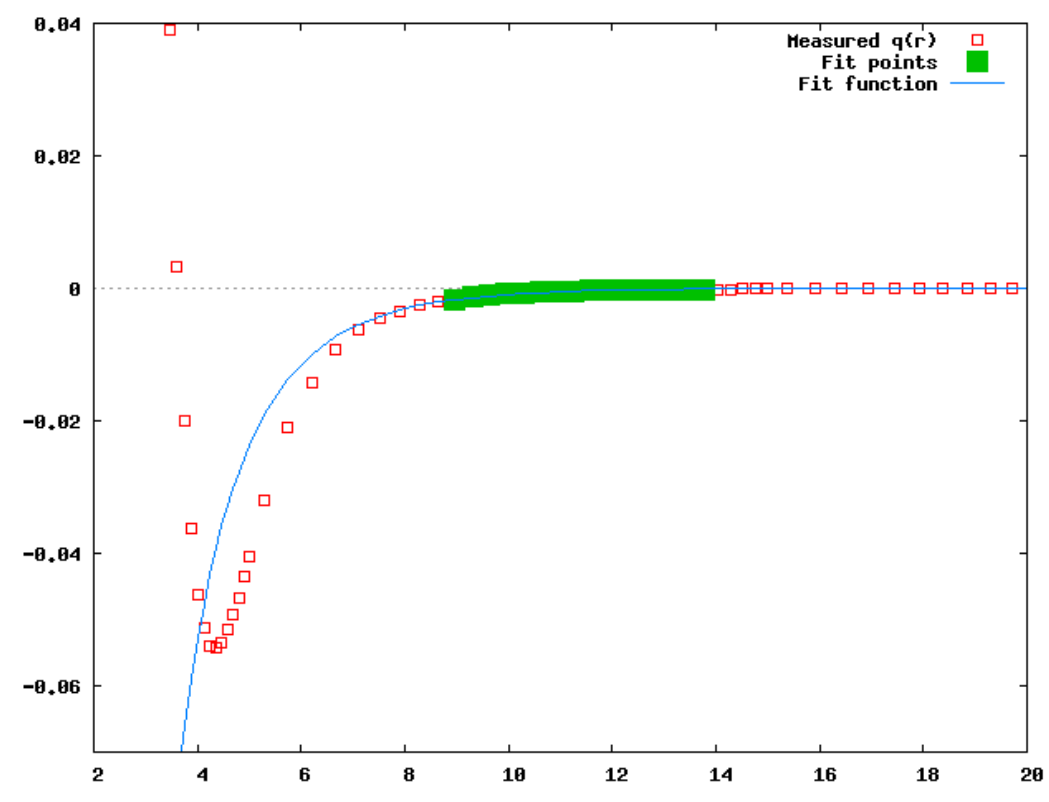

FIG. 1. The density of the topological susceptibility $\chi(r) \sim\langle q(r), q(0)\rangle$ as function of separation $r$ such that $\chi \equiv \int d r \chi(r)$, adapted from [60]. Plot explicitly shows the presence of the contact term with the "wrong sign" (narrow peak around $r \simeq 0$ ).

We reproduce Fig.1 from ref.[60] to illustrate few elements which are crucial for this work and which are explicitly present on the plot. First of all, there is a narrow peak around $r \simeq 0$ with a "wrong sign". Second, one can observe a smooth behaviour in extended region of $r \sim$ few fm with the opposite sign. Both these elements are present in the lattice computations as one can see from Fig.1. The same important elements are also present in our ghost's based computations given by eq. (19). In different words, the Veneziano ghost does model the crucial property of the topological susceptibility related to summation over topological sectors in gauge theories. This feature can not be accommodated by any physical asymptotic states as it is related to non-dispersive contribution with "wrong sign" as explained in the text. Furthermore, as non-dispersive positive contribution has singular behaviour at the core, this behaviour can not be reproduced by any finite size pseuodoparticles such as finite size instantons in instanton liquid model [61], see more comments on this matter in section III C.

Most important lesson from these discussions is that the lattice simulations unambiguously show that the low energy relations discussed in section II A are not saturated by physical asymptotic states. Therefore, the effective potential derived in Section II B and representing these low energy relations also can not be unambiguously associated with some physical propagating degrees of freedom (or any their combinations). In different words, the axion field $a$ is not expandable in terms of some asymptotic states (pseudoscalar glueballs), and can not be described with a canonical kinetic term. Though these fields can not be associated with propagating degrees of freedom, the corresponding "non-dispersive" fluctuations are obviously present in lattice Monte Carlo simulations [56-60].

In what follows we attempt to identify the relevant gauge configurations which make crucial contributions to the low energy relations (16) and (17). The most important observation from previous section II B is that the $2 \pi$ periodicity of the effective potential hints on possible Euclidean configurations in form of the domain walls which potentially can contribute into the non-dispersive term (17). In fact, the lattice results to be reviewed below apparently suggest that such long range structure indeed emerges, and observed configurations indeed make crucial contribution to the low energy relations (16) and (17). However, the corresponding configurations can not be easily described in terms of conventional quantum field theory (QFT) degrees of freedom. Still, one can argue that the gauge configurations can be described in terms of the dual holographic description when the relevant Euclidean configurations can be identified with so-called D2 branes [55, 67-69]. The only element which is important for us in what follows is that the tension of these D2 branes vanishes below the QCD phase transition $T<T_{c}$ such that an arbitrary large number of these objects can be formed ${ }^{4}$.

\footnotetext{
${ }^{4}$ Vanishing tension in the dual description in the confined phase is a result of the Hawking-Page phase transition [70] when the D2 brane shrinks to the tip of a cigar type geometry. It can be interpreted as an instability of a solution. It can be also interpreted as a formation of tensionless objects. The last interpretation is adapted in the present work. While there are some hints from QFT viewpoint on presence of Euclidean domain wall configurations in the system as we discussed in section II B, it is very difficult to describe them in terms of conventional QFT degrees of freedom.
} 


\section{B. Insights from numerical lattice simulations and holographic description: the emergence of coherent structures}

The recent Monte Carlo studies of pure glue gauge theory have revealed a laminar structure in the vacuum consisting of extended, thin, coherent, locally low-dimensional sheets of topological charge embedded in $4 \mathrm{~d}$ space, with opposite sign sheets interleaved, see original QCD lattice results [56, 63-65]. A similar structure has been also observed in QCD by a different group [57] and also in two dimensional $C P^{N-1}$ model [66]. Furthermore, the studies of localization properties of Dirac eigenmodes have also shown evidence for the delocalization of low-lying modes on effectively low-dimensional surfaces. It is not a goal of the present paper to cover this subject with a number of subtle points which accompany it. Instead, we limit ourselves by mentioning very few key properties of the gauge configurations which apparently make crucial contributions to the low energy relations (16) and (17). Here is the (incomplete) list of the unusual features of these gauge configurations:

a) The tension of the "low dimensional objects" vanishes below the critical temperature and these objects percolate through the vacuum, forming a kind of a vacuum condensate;

b) These "objects" do not percolate through the whole $4 \mathrm{~d}$ volume, but rather, lie on low dimensional surfaces $1 \leq d<4[64]$

c) The total area of the surfaces is dominated by a single percolating cluster of "low dimensional object";

d) The contribution of the percolating objects to the gluon condensate and therefore to correlation function (16) is opposite in sign compared to its total value;

e) The contribution of the percolating objects to the topological susceptibility (17) has the same sign compared to its total value;

f) The width of the percolating objects apparently vanish in the continuum limit similar to narrow peak of the lattice size around $r \simeq 0$ plotted on Fig. 1 .

It is very difficult to understand all those properties using conventional quantum field theory analysis. At the same time, using the holographic description the interpretation of these Monte Carlo results fits very nicely with conjecture that the observed structure can be identified with the D2 branes in holographic description[55, 67-69].

In particular the tension of the D2 branes vanishes in confined phase as a result of cigar geometry in the holographic $5 \mathrm{~d}$ description such that the $\mathrm{D} 2$ brane shrinks to the tip as we already mentioned. Therefore, an arbitrary large number of D2 branes could be produced, resulting in their condensation. The observed percolation (condensation) of these objects in lattice simulations unambiguously imply that they must have vanishing effective tension. Otherwise, only finite, not infinite percolating clusters could be observed, in contrast with observations listed as item c). In Minkowski space-time these Euclidean $4 \mathrm{~d}$ objects can be thought as tunnelling processes which are taking place in vacuum.

Other properties listed above are also have simple and natural interpretation within holographic framework representing the extended, thin, coherent objects as D2 branes. In particular, numerical observation listed as item e) above, reveals that the contribution of the D2 branes into the topological susceptibility $\chi_{E u c l}$ given by eq. (17) has the same positive sign as the contact term itself. Furthermore, both contributions have opposite sign in comparison with any propagating physical states (18). In different words, lattice studies are consistent with non-dispersive nature of these objects.

Another numerical observation listed as item d) above, reveals that the contribution of the D2 branes to the correlation function (16) has the positive sign which is the opposite to the total value of the correlation function (16) which is negative. The presence of this positive contribution indicates that there is a non-dispersive portion in (16). This feature implies that there is some ambiguity in standard procedure to saturate the low energy theorem (16) by physical propagating asymptotic states.

Few comments are in order on interpretation of the D2 branes as Euclidean objects describing the tunnelling processes between degenerate states, see [55] for the detail discussions. The term "degeneracy" should not be confused with conventional term "degeneracy" when two or more physically distinct states are present in the system. In the context of this paper the "degeneracy" implies there existence of winding states $|n\rangle$ constructed as follows: $\mathcal{T}|n\rangle=|n+1\rangle$. In this formula the operator $\mathcal{T}$ is the large gauge transformation operator which commutes with the Hamiltonian $[\mathcal{T}, H]=0$ which results in "degeneracy" of the winding states $|n\rangle$. The presence of $n$ different sectors in the system is reflected by summation over $n$ in path integral approach. The property $\mathcal{T}|n\rangle=|n+1\rangle$ is analogous to the Bloch's system in condensed matter physics with the "only" difference that the states $|n\rangle$ are not physically distinct states, and there is no any real "physical" degeneracy in the system. The physical vacuum state in QCD is unique and constructed as a superposition of $|n\rangle$ states. This interpretation explains the "wrong" sign in residues of the correlation function (17) in comparison with conventional formula (18) as we describe the tunnelling of the Euclidean D2 objects between winding states $|n\rangle$ rather than the tunnelling of conventional physical particles between distinct vacuum states in condensed matter physics in the Bloch's case, see [55] for the detail discussions.

Such a tunnel-based interpretation of D2 branes is consistent with representation of the contact term (which we assume is saturated by the D2 branes) as a total divergence. Indeed, the non-dispersive contact term with "wrong 
sign" in topological susceptibility (19) can be represented as a surface integral

$$
\begin{aligned}
\chi_{Y M} & \sim \int d^{4} x\left[\delta^{4}(x)\right]=\int d^{4} x \partial_{\mu}\left(\frac{x^{\mu}}{2 \pi^{2} x^{4}}\right)=\oint_{S_{3}} d \sigma_{\mu}\left(\frac{x^{\mu}}{2 \pi^{2} x^{4}}\right) \\
& =\frac{1}{24 \pi^{2}} \oint_{S_{3}} d \sigma_{\mu} \epsilon^{\mu \nu \lambda \sigma} \operatorname{Tr}\left[U \partial_{\nu} U^{-1} U \partial_{\lambda} U^{-1} U \partial_{\sigma} U^{-1}\right]
\end{aligned}
$$

where matrix $U$ describes the topologically non-trivial gauge configurations interpolating between two different winding states $|n\rangle$ and $|n+1\rangle$. Such a representation (20) of the non-dispersive term in topological susceptibility (19) again supports the tunnelling interpretation of the contact term. The dimensional parameter $\sim f_{\eta^{\prime}}^{2} m_{\eta^{\prime}}^{2}$ in eq. (19) should be interpreted as a number of tunnelling events per unit time per unit $3 \mathrm{~d}$ volume in the vacuum. This coefficient can be computed precisely in the so-called deformed QCD where all calculations are under complete theoretical control in weakly coupled regime, and where such interpretation becomes precise and unambiguous statement [46]. It is important to emphasize that the singular behaviour of the contact term $\sim \delta^{4}(x)$ is not an artifact of any approximations, in spite of the fact that this behaviour was established in simplified models: in the "deformed QCD" [46] and in the Veneziano model (19). This singular behaviour and the vanishing width (in continuum limit) of the contact term have been observed in the lattice simulations [56-60].

Also, the lattice measurements are also consistent with vanishing width of these "objects", see item $\mathrm{f}$ ) from the list above. These measurements, again, support the tunnelling interpretation of the contact term. Indeed, it is well known property of conventional quantum mechanics that the individual photons penetrate an optical tunnel barrier with an effective group velocity considerably greater than the vacuum speed of light [71], see also review [72]. In different words, the tunnelling time can not be distinguished (experimentally) from zero. In quantum field theory context it means that the configurations responsible for tunnelling events could have vanishing size (in continuum), which is precisely what has been measured on the lattices, see item f) above. The same feature can be also seen on Fig. 1 where the contact term with vanishing size (in continuum) has a "wrong sign" in Euclidean lattice simulations. This feature again supports the tunnelling interpretation of this term in Minkowski space.

\section{Few more remarks on long range structure}

With the structure just described the following question immediately emerges: what is the relation between "extended, thin, coherent, locally low-dimensional sheets" and other well-known topological objects e.g. instantons from the instanton liquid mode (ILM) [61]? Instantons from ILM are finite size objects, have been studied on the lattices, and is known to give a considerable contribution to the low energy relations (16) and (17). The crucial difference is that ILM has inherent scale, the instanton size such that all correlations decay on this scale $\sim 0.5$ fm. This scale enters all computations, including density-density correlation function shown on Fig. 1. The corresponding positive contribution to the peak would have a width of order the size of the instanton in ILM in contrast with vanishing size of the core observed in unbiased lattice simulations [56-60]. These lattice studies were based on new computational technique when no any auxiliary procedures such as cooling/smoothing/smearing are used in analysis. At the same time, previously used technique when cooling/smoothing/smearing was unavoidable part of the analysis is known to be strongly biased towards classical solutions. In fact, the original papers $[63,64]$ were precisely devoted to this question with the main conclusion is that the topological charge density distribution is not localized in any well -defined finite size lumps, but rather it is strongly delocalized in form of low dimensional objects as discussed above. To conclude: our viewpoint is that ILM (or any other model based on classical finite size objects) may provide a good estimate for some integral characteristics which are not sensitive to the fine details of topological charge density distribution. Such models would fail in description of some other effects which are sensitive to local distribution of the topological density and its specific features such as long range order.

Our next comment is as follows. The discussions presented above suggest that the relevant structure can not be described in terms of semiclassical configurations with finite size and tension (such as instantons/sphalerons etc). Can one visualize the relevant objects discussed above in section III B, at least qualitatively? In fact, an analogous domain wall structure is known to exist in QFT at large temperature in weak coupling regime where it can be described in terms of classical equation of motion. These are so-called $Z_{N}$ domain walls which separate domains characterized by a different value for the Polyakov loop at high temperature. As is known, see e.g. recent review paper [73] and references therein, these $Z_{N}$ domain walls interpolate between topologically different but physically identical states connected by large gauge transformations similar to our discussions at the end of section II B. At high temperature these objects can be described in terms of classical equation of motion. In this regime they have finite tension $\sim T^{3}$ such that their contribution to path integral is strongly suppressed. While the corresponding topological sectors are still present in the system at low temperature (though they are realized in a different way) it is not known how to 
describe the fate of $Z_{N}$ walls within QFT in strong coupling regime when semiclassical approximation breaks down. From holographic perspective however, the corresponding domain walls can be identified with tensionless D2 branes, and the hope is that such objects can be understood within holographic description as sketched in section IIIB.

\section{APPLICATIONS: FROM HEAVY ION COLLISIONS TO COSMOLOGY}

\section{A. Basic idea}

In previous sections II B and III we presented a number of arguments suggesting that the low energy relations (16), (17), and therefore the low energy potential (15), are saturated by coherent, extended, thin gauge configurations. The arguments were based on recent lattice results [56, 57, 63-65] and some model considerations. We interpreted these configurations as tunnelling processes which are happening all the time in Minkowski vacuum with no interruptions [55]. These tunnelling processes in Minkowski vacuum (when no any external sources are present in the system) do not lead to any emission or absorption of real particles, similar to the persistent tunnelling events in Bloch's case. These tunnelling events simply select an appropriate ground state of the system which is a specific superposition of $|n\rangle$ states. In what follows we assume that these coherent, gauge fluctuations saturate the low energy relations, and we concentrate only on these configurations. As we shall see below, the long range structure of the vacuum fluctuations plays a crucial role in both applications considered below. If this structure were not present in the system, both effects would be negligibly small. In different words, all conventional models with finite correlation length would predict a negligibly small magnitude for the effects considered below. The long range order advocated in this work is the key element fundamentally different from all other models characterized by finite correlation length as discussed in section III C.

The effective potential (15) which gives us a hint that such long range order is present in the system (as a result of $2 \pi$ periodicity of the potential supporting the long range domain walls) in this set up should be interpreted as follows. While each gauge configuration has definite sign of the topological charge density, the opposite sign sheets alternate. This delicate cancellation between the opposite sign sheets leads to the known result corresponding to minimization of the effective potential in physical vacuum: $\langle a\rangle_{\min }=0,\langle\eta\rangle_{\min }=0$. This is exactly the result obtained by conventional integrating out $G^{2}$ and $G \tilde{G}$ fields as described in section II B at $\theta=0$. In different words, the total vacuum expectation value of $\mathcal{P}$-odd operator $\langle a\rangle \sim\langle G \tilde{G}\rangle=0$ vanishes as it should at $\theta=0$.

However, when some external sources are present in the system a delicate cancellation between the opposite sign sheets may lead, in general, to a local minimum with nontrivial values for the dilaton and the axion fields in the region where external impact is felt, i.e. $\langle a(x)\rangle \neq 0,\langle\eta(x)\rangle \neq 0$. Non-vanishing and long range correlated $\langle a(x)\rangle \neq 0$ obviously implies that $\langle G \tilde{G}\rangle \neq 0$ in the same region of space-time. Indeed, the axion field $\langle a\rangle \neq 0$ plays the role of effective $\theta_{\text {ind }} \neq 0$ as can be seen from definition (10). Precisely this deviation from vacuum values (14), as we shall argue below, will be the main source for the local violation of $\mathcal{P}$ invariance in QCD as well as for the "apparent universal thermalization" in high energy collisions. The long range order advocated in this work plays a crucial role is estimation of a magnitude of the effects. All conventional models based on instantons/sphalerons with finite correlation length would lead to negligible effects.

We consider two applications of these ideas, which are separated by gigantic differences in scales. First, in section IV B we consider high energy collisions when Minkowski vacuum is disturbed with a typical $\Lambda_{Q C D} \sim 10^{2}$ MeV scale. Secondly, in section IV C we consider cosmology when the deviation from Minkowski vacuum is characterized by the Hubble constant $H \sim 10^{-33} \mathrm{eV}$. These two applications with drastically different scales have a unique crucial element in common: in both cases the Minkowski vacuum is deviated from its equilibrium dynamics, and the observable effects are proportional to this deviation. This disturbance, of course, is numerically very different: in first case the external influence leads to the effects of order of one, while in the second case the effect is $\sim H / \Lambda_{Q C D} \sim 10^{-41}$. Still, in both cases, all observational effects are due to the same "non- cancellation" between long range vacuum configurations with opposite sign sheets interleaved. This "non-cancellation" in both cases is a direct consequence of an external disturbance of the vacuum from its perfect state $\langle a\rangle=0,\langle\eta\rangle=0$ in infinitely large Minkowski space time. Normally, in unperturbed vacuum, these long range vacuum configurations are subject of delicate and exact cancellation between low-dimensional opposite sign sheets of topological charge. This exact cancellation leads to $\langle G \tilde{G}\rangle=0$ in the vacuum state. Our key assumption is that this exact cancellation can not been maintained in new environments considered below in sections IV B and IV C. Furthermore, this "non-cancellation" occurs in extended region of space-time with the correlation length being the same order of magnitude as size of vacuum sheet itself, which is exactly the source of long range effects discussed below. 


\section{B. Terrestrial applications: high energy collisions at RHIC and LHC.}

In the framework presented in sections II B and III we argued that the long range correlated, thin gauge configurations with vanishing width make a crucial contribution to the low energy relations (16), (17), and therefore to the low energy potential (15). In Minkowski space time the Euclidean objects with vanishing sizes should be interpreted as the tunnelling events which are happening with superluminal velocity, see last paragraph in section III. Now we present some arguments suggesting that precisely these type of configurations with vanishing width (in Euclidean space) and interpreted as instantaneous superluminal tunnelling events (in Minkowski space) are responsible for the origin of local violation of $\mathcal{P}$ invariance as well as for the "apparently" thermal spectrum in high energy collisions.

The basic picture behind this proposal is that high energy collisions including $e^{+} e^{-}, p p$ and $p \bar{p}$ interactions do not completely destroy the coherent vacuum structure described in section III. Instead, the collisions lead to some shifts and distortions between low-dimensional sheets (which existed before the collision) rather than breaking them. These distortions, however, spoil the exact cancellations between the long range vacuum configurations which existed in vacuum before the collision occurred. It is crucial that these Euclidean long range vacuum configurations with vanishing width actually describe instantaneous tunnelling events in Minkowski space. Distortion of these configurations leads to emission of real physical particles. It is similar to the inelastic scattering processes of quasi-electrons in the presence of impurities distorting the perfect lattice structure in Bloch's case in condensed matter physics. In this proposal two crucial puzzles formulated in section 2 are immediately resolved as follows:

a) The thermal spectrum in $e^{+} e^{-}, p p$ and $p \bar{p}$ high energy collisions emerges in spite of the fact that the statistical thermalization could never be reached in those systems. This is due to the fact that this spectrum is a result of the same tunnelling processes which have been happening all the time even before the collision occurs. However, in the vacuum these tunnelling processes (between "degenerate" $|n\rangle$ states) do not lead to any emissions as a result of the delicate cancellations which is a consequence of exact symmetry $[\mathcal{T}, H]=0$. The collision slightly disturbs the vacuum and leads to formation of a locally different $\left|\theta_{\text {ind }}\right\rangle=\sum \exp \left(i n \theta_{\text {ind }}\right)|n\rangle$ states with quasi-momentum $\theta_{\text {ind }}$ constructed from the same "degenerate" and correlated $|n\rangle$ states. These $\left|\theta_{\text {ind }}\right\rangle$ states decay as a result of tunnelling processes leading to the thermal spectrum of emitted particles. The spectrum could be nothing else but thermal as it is entirely due to the tunnelling events with superluminal velocity when information can not be transferred ${ }^{5}$. In holographic picture, the observed spectrum is a result of emissions from fluctuating D2 branes which are disturbed by collisions.

We must emphasize that we concentrate on low energy part of the spectra in this paper; the high part comes from many different other processes which shall not be even mentioned here. Furthermore, in heavy ion collisions a conventional statistical thermalization is achieved, in contrast with "apparent thermalization" discussed in this work. This part of the spectra, being characterized by a different so-called saturation $Q_{\text {sat }}$ scale, is also not a part of consideration of the present work.

b) An approximate universality of the temperature with no dependence on energy of colliding particles nor their nature (including $e^{+} e^{-}, p p$ and $p \bar{p}$ collisions) is due to the fact that the emission occurs from the distorted QCD vacuum state represented by the long range vacuum configurations with vanishing width rather than from the colliding particles themselves. In holographic picture the observed spectrum is a result of emissions from the disturbed D2 branes; therefore, it can not be sensitive to a nature of disturbance and always remains the same. The intensity of the emission, of course, depends on the nature of colliding particles, and total energy being transferred to the D2 branes to excite them from their normal equilibrium state in Minkowski space-time.

Along with this simple resolution of two aforementioned puzzles a) and b), this proposal shed some light on another puzzle formulated in section I A: why the $\mathcal{P}$ odd domains are so large, much larger than conventional $\Lambda_{Q C D}^{-1}$ scale? Resolution of this puzzle as given in item c) below also elucidates the reason why we treat two naively different problems formulated in sections I A and IB as two sides of the same coin.

c) A puzzle with a long- range structure of the $\mathcal{P}$ odd domains within our framework is resolved as follows. The low-dimensional coherent sheets responsible for the tunnelling as explained above, carry the quantum numbers of the topological charge density $G \tilde{G}$ which is $\mathcal{P}$ and $\mathcal{C P}$ odd operator. In fact, it was exactly this feature which was studied on the lattices $[56,57,63-65]$. Therefore, the distortion of these low-dimensional coherent sheets due to the collisions lead to a local "non-cancellation" between different low-dimensional coherent sheets. Precisely this structure becomes coherent on the large scales, much larger than $\Lambda_{Q C D}^{-1}$ as a result of collisions. In different words, the collisions do not produce a coherent large $\mathcal{P}$ odd domain. Rather, the collisions do not completely destroy the coherent structure which always existed in vacuum. The role of collisions in this framework is that the collisions slightly destroy the delicate cancellation which is inherent feature of the perfect undisturbed vacuum state as was discussed in section IV A.

\footnotetext{
5 It is interesting to note that the computations based on the Euclidean configurations, e.g. instantons, describing the tunnelling events, also produce a Planck like spectrum[74-76]. We interpret this result as a generic feature of physics of tunnelling rather than a simple coincidence.
} 

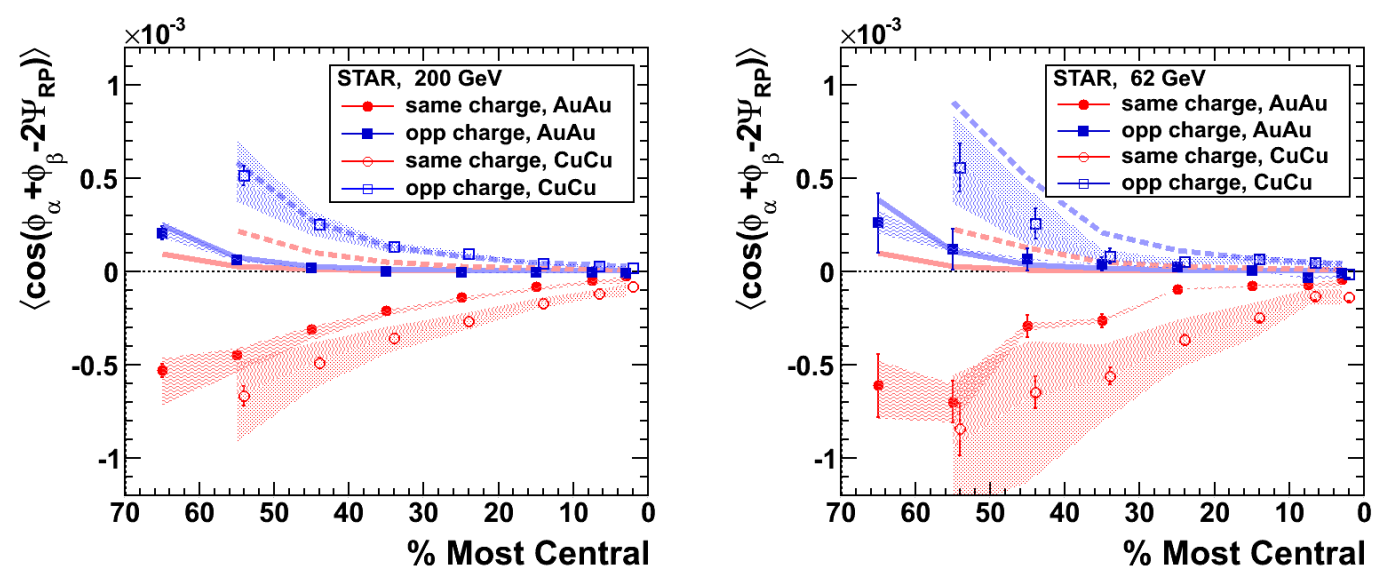

FIG. 2. Data for $\sqrt{s_{N N}}=200 \mathrm{GeV}$ and $\sqrt{s_{N N}}=62 \mathrm{GeV}$ for $\mathrm{Au}+\mathrm{Au}$ and $\mathrm{Cu}+\mathrm{Cu}$ collisions (adapted from [2]). The plots demonstrate the universality in behaviour, see items d) and e) in the text for the details.

This picture gives a precise dynamical realization of the conjecture formulated in [9] that these two phenomena outlined in sections I A and IB are in fact are intimately related as both originated from the dynamics of the coherent vacuum structure observed on the lattices and described in section III. The crucial point here is this: though we can not presently compute the spectrum, it must demonstrate the same features for $\mathcal{P}$ even as well as for $\mathcal{P}$ odd correlations with universal Planck spectrum observed in all high energy collisions. Some supporting evidence for this behaviour is listed in items $\mathrm{f}$ ), $\mathrm{g}$ ) $\mathrm{h}$ ) below. In holographic description both these phenomena formulated in sections I A and IB are due to the same tunnelling events described by D2 branes which emit real particles as a result of small disturbance of the vacuum state resulted from the collision.

With this basic picture outlined above the main question is: What are the technical tools to describe these effects quantitatively? As we argued in section III C the relevant structure can not be described in terms of semiclassical physics with finite correlation length using ILM or sphalerons as relevant pseudoparticles. Instead, one should study the dynamics using the dual holographic description. To be more specific, one should analyze the dynamics of tensionless D2 branes when they are slightly disturbed by external forces (including the fluctuations of D2 branes leading to the emission of real particles). In lattice simulations, the relevant information is hidden in dynamics of extended coherent vacuum sheets when they are slightly disturbed by the collisions. The fluctuations of the corresponding correlated coherent objects are expected to emit particles with thermal spectrum. In principle, these ideas can be tested using the lattice simulations. These are technically very challenging problems, which are beyond the scope of the present work.

Still, there are some model independent consequences of this framework which are based exclusively on the assumption that the collisions do not completely destroy the coherent structure, but rather slightly disturb it. These generic consequences will be listed below. We leave the subject with more specific but model dependent calculations for a future work. Here we continue the list of model-independent consequences of this framework:

d) When a system is not infinite, but sufficiently large (e.g. large ions with size $L \gg \Lambda_{\text {QCD }}^{-1}$ ) the observable $\mathcal{P}$ odd effect due to the collisions of objects size $L$ are expected to be proportional to $1 / L^{p}$ with some power $p$. The $L^{-p}$ scaling essentially describes the deviation of the system from the ground state in the region $L$ as a result of collision. We refer to Appendix B where we present a number of arguments (including some QCD lattice results) supporting $L^{-p}$ scaling. Such a Casimir like behaviour should be contrasted with naively expected exponential suppression $\exp (-L)$ when a mass gap being inherent feature of QCD is present in the system. The crucial point is that $L^{-p}$ correction is originated from non-dispersive contributions which are not related to any physical states as discussed in section III.

e) As one can see from Fig. 2 some suppression of the measured correlations with increasing the size of the system indeed has been observed. We would like to interpret this suppression as a manifestation of the $L^{-p}$ scaling. Indeed, the effect for $\mathrm{Au}+\mathrm{Au}$ collisions with $A \simeq 197$ is obviously suppressed in comparison with $\mathrm{Cu}+\mathrm{Cu}$ collisions with $A \simeq 64$. There are many other effects which influence this ratio (both systems are obviously not very large when derivative expansion is justified). However, the effect goes in the right direction (the effect is stronger for a smaller size system $\mathrm{Cu}+\mathrm{Cu}$ than for a larger $\mathrm{Au}+\mathrm{Au}$ system).

f) The correlations due to the local $\mathcal{P}$ violation should demonstrate the universal behaviour similar to the "universal apparent thermalization" discussed in section IB as the source for the both effects is the same as argued in this paper. In particular, the effect should not depend on energy of colliding ions. Such independence on energy is indeed 


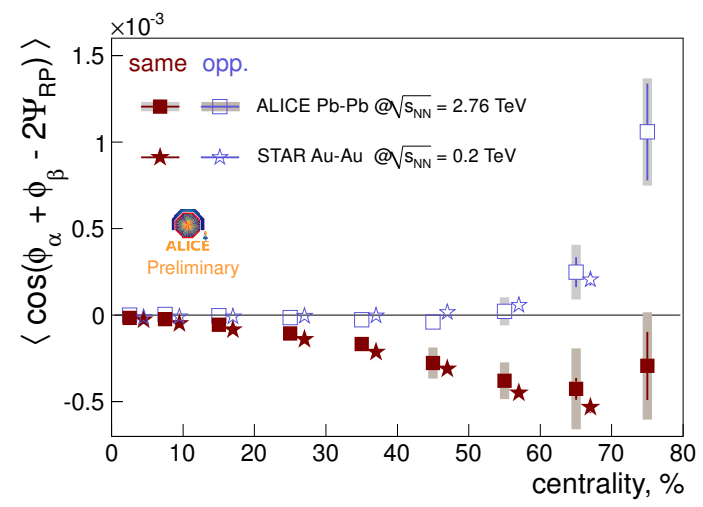

FIG. 3. Data for Pb-Pb collisions with ALICE at the LHC at $\sqrt{s_{N N}}=2.76 \mathrm{TeV}$ and $\mathrm{Au}+\mathrm{Au}$ collisions with STAR at RHIC at $\sqrt{s_{N N}}=0.2 \mathrm{TeV}$ (adapted from [4]). The plot demonstrates the universality in behaviour (energy independence, $2.76 \mathrm{TeV}$ vs $0.2 \mathrm{TeV}$ ), see item $\mathrm{g}$ ) in the text for the details.
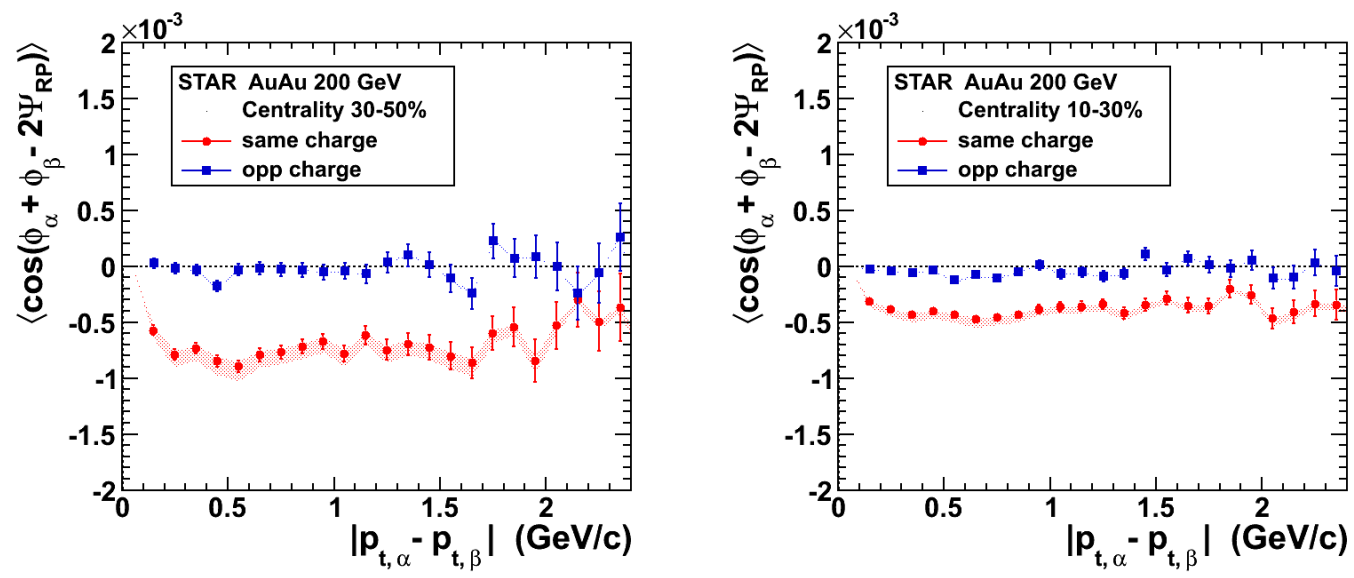

FIG. 4. Data for $\sqrt{s_{N N}}=200 \mathrm{GeV}$ and $\sqrt{s_{N N}}=62 \mathrm{GeV}$ for $\mathrm{Au}+\mathrm{Au}$ and $\mathrm{Cu}+\mathrm{Cu}$ collisions (adapted from [2]). The plots demonstrate the universality in behaviour and independence on $\left|k_{\perp, \alpha}-k_{\perp, \beta}\right|$, see item $\left.\mathrm{h}\right)$ in the text for the details.

supported by observations where correlations measured in $\mathrm{Au}+\mathrm{Au}$ and $\mathrm{Cu}+\mathrm{Cu}$ collisions at $\sqrt{s_{N N}}=62 \mathrm{GeV}$ and $\sqrt{s_{N N}}=200 \mathrm{GeV}$ are almost identical and independent on energy, see Fig. 2.

g) We expect the same tendency to continue for the LHC energies. In fact, in [9] we had predicted (before the LHC preliminary results $[3,4]$ have been posted) that the corresponding correlations at the LHC energies should demonstrate a similar strength and a similar features found at RHIC. Preliminary recent results from ALICE Collaboration indeed confirm this prediction. As one can see from Fig 3, the ALICE Pb-Pb results for 3-particle correlator at $\sqrt{s_{N N}}=2.76 \mathrm{TeV}$ are almost identically coincide with RHIC Au+Au results at $\sqrt{s_{N N}}=200 \mathrm{GeV}$, which is precisely what was anticipated as the sizes for $\mathrm{Au}^{79}$ and $\mathrm{Pb}^{82}$ are almost the same in contrast with much lighter and smaller $\mathrm{Cu}^{29}$ when effect should be stronger, see item e) above.

h) The arguments presented above on universality of the correlation strength do not depend on transverse momenta $k_{\perp}^{2}$. This is a consequence of the same universal behaviour discussed above. Indeed, the low-dimensional coherent sheets responsible for the tunnelling/emission are the $\mathcal{P}$ odd objects as they carry the quantum numbers of the topological charge density. Therefore, the corresponding emissions always contribute to the correlations presented on Fig. 4 with the same rate as for conventional Hagedorn emission. This consequence of the universality is also consistent with observations, see Fig 4 , where it is found that the correlation depends very weakly on $\left|k_{\perp, \alpha}-k_{\perp, \beta}\right|$. The signal (intensity of the asymmetry) shown in Fig. 4 obviously becomes weaker for central collisions, as $\mathcal{P}$ odd correlations are washed out for the central collisions, see discussions below on relevant time scales for this problem. However, the dependence on transverse momenta $k_{\perp}^{2}$ is determined by the same tunnelling processes leading to the Planck spectrum, and therefore must be universal. We should emphasize once again that we are talking about 
the low energy part of the spectra where our consideration is applicable, see the corresponding comment in item a) above.

Few more comments. The qualitative consequences which follow from the picture outlined above are consistent with all presently available data. Even more that that, few predictions we had made in [9] have been recently confirmed at the LHC energies, see item g). The basic idea is that all effects are proportional to the deviations of the axion $a(x)$ and $\eta(x)$ fields from their vacuum values computed in infinitely large space-time (14). Small disturbances resulting from collision can be parametrized by $\left|\left\langle\partial_{\mu} a(x)\right\rangle\right| \sim\left|\left\langle\partial_{\mu} \eta(x)\right\rangle\right| \sim L^{-1}$ which is a measure of deviation from unperturbed ground state in the large region of size $L$. The deviations should be computed from their vacuum values: $\langle a\rangle=\theta / N_{c},\langle\eta\rangle=0$ presented in eq.(14). In a simplest case of slow time- dependent and spatially- independent variation of the axion and dilaton fields $a(t), \eta(t)$, the key parameter is the acceleration $|\langle\dot{a}\rangle| \sim|\langle\dot{\eta}\rangle| \sim|\mathbf{a}|$, see Appendix A. Derivative expansion which is assumed in this work can be justified only for small acceleration $\mathbf{a} \ll \Lambda_{\mathrm{QCD}}$.

In reality $\mathbf{a}$ is not numerically small number as estimations of ref. [8] suggest. Furthermore, the size of $\mathcal{P}$ odd domain can not be very large even for non-central collisions for physically available ions. The finite size effects and other non-universal features may lead to some corrections from the universal picture presented here. The crucial element for analysis of these non-universal features is the understanding of all relevant time scales of the problem. In particular, if for a given acceleration a a typical time scale $\Delta t$ for multiple emissions satisfies the condition $\Delta t \gg \mathbf{a}^{-1}$, there will be no any observable $\mathcal{P}$ odd effects as the system has time to completely adjust to a new environment caused by a collision ${ }^{6}$. To observe $\mathcal{P}$ odd effects the opposite condition $\Delta t \leq \mathbf{a}^{-1}$ must be satisfied. This hierarchy of scales also explains the dependence of strength of correlations from centrality as presented in Fig.2. Indeed, the peripheral collisions obviously correspond to a smaller disturbance of the ground state and correspondingly smaller acceleration a, while $\Delta t \sim \Lambda_{Q C D}^{-1}$ is essentially determined by conventional QCD scale and does not much depend on centrality. This is precisely the reason why the condition $\Delta t \leq \mathbf{a}^{-1}$ is likely to be met in heavy ion peripheral collisions. Strength of the correlation obviously increases with centrality where acceleration a decreases, in accordance with data presented in Fig.2.

The condition $\Delta t \leq \mathbf{a}^{-1}$ is less likely to be satisfied for centralities $(0 \%-5 \%)$ in heavy ion collisions as well as for $e^{+} e^{-}, p p$ and $p \bar{p}$ collisions, see [9] with more discussions on this point. In this case, one should expect a conventional Planck emission from disturbed low-dimensional extended coherent topological sheets as usual. However, the asymmetry presented in Figs. 2, 3 in this case will be largely washed out because too many disturbed sheets contribute to the emission with opposite signs.

Also, when total energy becomes sufficiently small, a typical momentum transfer $\Delta q$ of emitting particles is also getting smaller. It leads to increasing of typical $\Delta t \sim(\Delta q)^{-1}$ such that condition $\Delta t \leq \mathbf{a}^{-1}$ is no longer satisfied, and effect is washed out again. This time it is washed out not because of sufficiently large a, typical for centralities $(0 \%-5 \%)$, but rather because of relatively large $\Delta t \sim(\Delta q)^{-1}$ as a result of relatively small momentum transfer $\Delta q$.

Order of magnitude estimate. We are now in position to give a simple, order of magnitude, estimation for all $\mathcal{P}$ odd effects when all deviations from the vacuum are small, $|\langle\dot{a}\rangle| \sim|\mathbf{a}|$, see Appendix A. The simplest way to proceed is to use the description in terms of the Veneziano ghost which effectively describes the dynamics of the degenerate topological sector of QCD. The number density of the $\mathcal{P}$ odd domains with size $\lambda \simeq \frac{2 \pi}{\omega}$ can be estimated as follows, see [9] for the details

$$
d N_{\omega}=\frac{d^{3} k}{(2 \pi)^{3}} \frac{2}{\left(e^{2 \pi \omega / \mathbf{a}}-1\right)} .
$$

The total contribution to the energy associated with these soft fluctuations is

$$
E_{\text {ghost }} \simeq \int \frac{d^{3} k}{(2 \pi)^{3}} \frac{2 \omega}{\left(e^{2 \pi \omega / \mathbf{a}}-1\right)}=\frac{\pi^{2}}{15}\left(\frac{\mathbf{a}}{2 \pi}\right)^{4},
$$

which should be compared with conventional contribution due to $N_{f}$ massless quarks and $N_{c}^{2}-1$ gluons,

$$
E_{q+g} \simeq \frac{\pi^{2}}{15}\left(\frac{\mathbf{a}}{2 \pi}\right)^{4}\left[\left(N_{c}^{2}-1\right)+\frac{7 N_{c} N_{f}}{4}\right] .
$$

\footnotetext{
${ }^{6}$ It is similar to more familiar case of fluctuating instantons (sphalerons) and antiinstantons which are $\mathcal{P}$ odd objects themselves. However, these $\mathcal{P}$-odd fluctuations of the topological charge do not lead to any observable $\mathcal{P}$ odd effects because of the cancellations between small instantons and antiinstantons. Difference with our case is that the relevant objects in our analysis are strongly correlated, thin extended gauge configurations discussed in section III. It is precisely these long range correlations may lead to "non- cancellation" between two types of low-dimensional sheets observed on the lattices, and identified with D2 branes in holographic description.
} 
Therefore, the relative energy associated with slow ghost fluctuations with $0^{+-}$quantum numbers in comparison with conventional fluctuations of quarks and gluons with Hagedorn spectrum is estimated to be

$$
\kappa \equiv \frac{E_{\text {ghost }}}{E_{q+g}} \sim \frac{1}{\left[\left(N_{c}^{2}-1\right)+\frac{7 N_{c} N_{f}}{4}\right]},
$$

which is numerically $\sim 0.05$. The effect is parametrically small at large $N_{c}$ and proportional $\sim 1 / N_{c}^{2}$ which is a typical suppression for any phenomena related to topological fluctuations. The effects related to the disturbance of the well organized structure described in section III, obviously vanish at $\mathbf{a}=0$ as eq. (22) states. Non-vanishing $\mathbf{a} \neq 0$ effectively describes the dynamics of excited "topological sectors" as discussed in IV A. The factor $\kappa$ essentially counts number of fluctuating degrees of freedom which lead to the $\mathcal{P}$ and $\mathcal{C P}$ odd environment. However, these degrees of freedom are not the asymptotic states, and they do not propagate to infinity, and they do not contribute to the absorptive parts of any correlation functions. Rather, they contribute to the non-dispersive parts of the correlation functions as explained in section III A.

There are few more factors which must be present in estimate for the observed asymmetries presented on Figs. 2, 3, 4. First, there is a trivial numerical factor proportional to the electric charge $e \sim \sqrt{\alpha} \sim 10^{-1}$. Second, there is an additional suppression factor $\sim L^{-1}$ as a manifestation of the Casimir scaling discussed in the text and in Appendix B. This is the key element of the entire framework: all observed asymmetries would be much smaller if the long range order is not present in the system. We parametrize the Casimir behaviour $\sim L^{-1}$ as follows

$$
\begin{gathered}
\text { [asymmetries on Figs. 2, 3, 4] } \sim e \cdot \kappa \cdot \frac{f(\gamma)}{L \Lambda_{\mathrm{QCD}}} \sim 5 \cdot 10^{-4}, \\
\gamma \equiv \mathbf{a} \Delta t, \quad f(\gamma \ll 1) \simeq \text { const }, \quad f(\gamma \gg 1) \simeq 0,
\end{gathered}
$$

where for numerical estimates we assume $L \Lambda_{\mathrm{QCD}} \simeq 10$. Numerical estimation (25) is consistent with intensities of the observed asymmetries presented on Figs. 2, 3, 4. The dimensionless parameter $\gamma \equiv \mathbf{a} \Delta t$ is a convenient way to parametrize different time-scales discussed above. Function $f(\gamma)$ in expression (25) vanishes at large $\gamma \gg 1$ and approaches a constant at small $\gamma \ll 1$ as argued above. The dependence on centrality is effectively represented by variation of parameter a, and therefore $\gamma$. Function $f(\gamma)$ in expression (25) also depends on many other characteristics of the system such as charge/size of a nucleus, its induced magnetic field, and many other non-universal parameters. Evaluation of function $f(\gamma)$ is a hard problem which is beyond the scope of the present work. The corresponding computations would obviously require some specific model- dependent assumptions, while we attempt in this work to formulate some model-independent consequences of this framework (see items $\mathbf{a}-\mathbf{h}$ above) which are based exclusively on the basic fundamental principles formulated in the beginning of this section, and not on any additional modeldependent/non-controllable approximations.

If a long range order would not be present in the system (for example, we would use a finite size sphaleron transition to estimate the effect), we would get a strong suppression $\exp \left(-\Lambda_{\mathrm{QCD}} L\right)$ instead of power like suppression $\left(L \Lambda_{\mathrm{QCD}}\right)^{-1}$ from eq. (25). Such a strong $\exp \left(-\Lambda_{\mathrm{QCD}} L\right)$ suppression is a consequence of a finite correlation length $\left(\Lambda_{\mathrm{QCD}}\right)^{-1}$ typical for all conventional models. It should be contrasted with framework advocated in this work when the long range order is inherent feature of the system. Therefore, any estimates based on conventional finite size objects would unavoidably lead to an estimate which is $\exp \left(-\Lambda_{\mathrm{QCD}} L\right) \cdot\left(L \Lambda_{\mathrm{QCD}}\right) \sim\left(10^{-3}-10^{-4}\right)$ smaller than the observed values.

We should emphasize once again that all measurements are $\mathcal{P}$ even observables. Therefore, there are many background processes which considerably contribute to the effect [28-32]. If future studies of this effect nevertheless confirm that the $\mathrm{CME}$ /charge separation effect is a main source of the observed event-by-event fluctuations, we would like to interpret this result as a strong evidence for the long range order present in the system, as advocated in this proposal. Much work needs to be done before a qualitative picture sketched above becomes a quantitative description of the $\mathcal{P}$ and $\mathcal{C P}$ odd correlations observed at RHIC and LHC [1-4].

\section{Cosmological applications: dark energy and accelerated universe}

In this section we consider another application related to the deviation of the ground state from its constant values (14) as a result of expansion of our FLRW universe. This deviation is determined by the Hubble constant $H \simeq 10^{-33} \mathrm{eV}$ which replaces acceleration parameter $\mathbf{a} \simeq 10^{2} \mathrm{MeV}$ describing the deviation of the QCD ground state resulting from a collision, see previous section IV B. This study may have some profound consequences on our understanding of FLRW universe we live in. What is more remarkable is the fact that the fundamental cosmological ideas formulated below, in principle, can be experimentally tested in heavy ion collisions at RHIC and LHC where the required unusual environment can be produced. 
Non-dispersive contribution with a "wrong sign" in topological susceptibility (19) obviously implies, as eq. (6) states, that there is also some energy related to this contact term. This $\theta-$ dependent portion of the energy not related to any physical propagating degrees of freedom, is well established phenomenon and tested on the lattice, see Fig.1; it is not part of the debates. What is the part of the debates and speculations is the question on how this energy changes when background varies. In different words, the question is: how does the non-dispersive contribution to the $\theta$ - dependent portion of the energy vary when conventional Minkowski background is replaced by FLRW universe with the horizon size $L \sim H^{-1}$ determined by the Hubble constant $H$ ? Similar problem has been already discussed in section IV B, where we argued that the entire effect of the collisions can be described as the deviation from the ground state values (14) and must be proportional to $L^{-p}$ when disturbance is relatively small for sufficiently large $L$, see item d) in previous section.

The motivation for this question in the present context is different as there are no any external sources which may change the properties of the ground state, similar to collisions in section IV B. Instead, the deviation from solution (14) emerges as our universe is not static, but rather, expanding with the rate $H$. We adapt the paradigm that the relevant definition of the energy which enters the Einstein equations is the difference $\Delta E \equiv\left(E-E_{\text {Mink }}\right)$, similar to the well known Casimir effect when the observed energy is in fact a difference between the energy computed for a system with conducting boundaries and infinite Minkowski space. This is in fact the standard subtraction procedure which is normally used for description the horizon's thermodynamics $[77,78]$ as well as in a course of computations of different Green's function in a curved background by subtracting infinities originated from the flat space [35]. In the present context such a definition $\Delta E \equiv\left(E-E_{\text {Mink }}\right)$ for the vacuum energy for the first time was advocated in 1967 by Zeldovich [79] who argued that $\rho_{\mathrm{vac}} \sim G m_{p}^{6}$ with $m_{p}$ being the proton's mass, see also [48] for related references. In different words, the dark energy observed in our universe might be a result of mismatch between the QCD vacuum energy computed in slowly expanding universe with the expansion rate $H$ and the one which is computed in flat Minkowski space.

This is exactly the motivation for question formulated in the previous paragraph: how does $\Delta E$ scale with $H$ ? The difference $\Delta E$ must obviously vanish when $H \rightarrow 0$ as it corresponds to the transition to flat Minkowski space. How does it vanish? A naive expectation based on common sense suggests that $\Delta E \sim \exp \left(-\Lambda_{Q C D} / H\right) \sim \exp \left(-10^{41}\right)$ as QCD has a mass- gap $\sim \Lambda_{Q C D}$, and therefore, $\Delta E$ must not be sensitive to size of our universe ${ }^{7}$. Such a naive expectation formally follows from the dispersion relations similar to (18) which dictate that a sensitivity to very large distances must be exponentially suppressed when a mass gap is present in the system.

However, as we discussed at length in this paper, along with conventional dispersive contribution (18) there is also the non-dispersive contribution $(17,19)$ which plays a crucial role in the construction of the ground state $(14)$. This term, as explained in section III is not related to any propagating physical degrees of freedom, and may lead to a power like scaling $\Delta E \sim H+\mathcal{O}(H)^{2}$ rather than naively expected $\Delta E \sim \exp \left(-\Lambda_{Q C D} / H\right)$ behaviour.

The Casimir power like scaling $\Delta E \sim H+\mathcal{O}(H)^{2}$ in QCD, if confirmed by future analytical and numerical studies, may have profound consequences for understanding of the expanding FLRW universe we live in. If true, the difference between two metrics (FRLW and Minkowski) would lead to an estimate

$$
\Delta E \sim H \Lambda_{Q C D}^{3} \sim\left(10^{-3} \mathrm{eV}\right)^{4},
$$

which is amazingly close to the observed DE value today without adjusting of any parameters. Such a behaviour $\Delta E \sim H+\mathcal{O}(H)^{2}$ was postulated in [80]. The power like scaling has received recently a solid theoretical and numerical support, see Appendix B for references and the details.

It is interesting to note that expression (26) reduces to Zeldovich's [79] formula $\rho_{\mathrm{vac}} \sim G m_{p}^{6}$ if one replaces $\Lambda_{Q C D} \rightarrow m_{p}$ and $H \rightarrow G \Lambda_{Q C D}^{3}$. The last step follows from the solution of the Friedman equation

$$
H^{2}=\frac{8 \pi G}{3}\left(\rho_{D E}+\rho_{M}\right), \quad \rho_{D E} \sim H \Lambda_{Q C D}^{3}, \quad \rho_{c}=\frac{3 H^{2}}{8 \pi G}
$$

when the DE component dominates the matter component, $\rho_{D E} \gg \rho_{M}$. In this case the evolution of the universe approaches a de-Sitter state with constant expansion rate $H \sim G \Lambda_{Q C D}^{3}$ as follows from (27).

One should add that a number of other fine tuning issues which always plague dark energy models, such as "coincidence problems", "drastic separation of scales", "unnatural weakness of interactions", etc., possess a simple and natural explanation within this framework without a single new field/coupling constant in the fundamental Lagrangian of Standard Model. In particular, the fine tuning problem which goes under the name of "cosmic coincidence" problem

\footnotetext{
7 Here and in what follows we emphasize on the power like sensitivity to arbitrary large distances irrespectively to their nature. In different words, a crucial distinct feature which characterizes the system we are interested in is the presence of dimensional parameter $L \sim H^{-1}$ in a system which discriminates it from infinitely large Minkowski space-time. For purposes of this work we do not discriminate the horizon size $H^{-1}$ of expanding universe from size $L \sim 10 \mathrm{fm}$ of a compact manifold we used in previous section IV B.
} 
finds its natural resolution as follows. The vacuum energy which attributed to the extra Casimir -type contribution (26) in our framework becomes relevant when its energy is of the same order of magnitude as the critical density, $\rho_{D E} \simeq \rho_{c}$. Equating these two quantities returns $t_{0} \sim H^{-1} \sim\left(G \Lambda_{Q C D}^{3}\right)^{-1} \sim 10$ Gyr, see eq. (27). This is indeed a correct estimate for the lifetime of the present universe.

To conclude this section: the key element for both applications considered in sections IV B and IV C is the paradigm that the observed effects are due to the disturbances of the ground state (14). The sources for these perturbations, of course, are very different: in first case it is a result of heavy ion collision, while in the second case it is a result of expansion of the universe. However, in both cases this deviation from uniform solution (14) demonstrates a Casimir power like correction to the vacuum energy in spite of the fact that QCD is a confined theory with a gap. Formally, the Casimir type behaviour emerges from non-dispersive contact term which is not related to any propagating degrees of freedom, but rather, originated from degenerate topological sectors of the theory. Therefore, a standard argument based on dispersion relations suggesting the exponentially weak sensitivity to arbitrary large distances is simply not applicable in this case. Microscopically this long-range order emerges as a result of dynamics of low-dimensional coherent sheets of gauge configurations seen in the lattice simulations and discussed in section III. We refer to Appendix $\mathrm{B}$ with more insights and discussions on this very nontrivial Casimir-like behaviour.

\section{CONCLUSION}

The main results of this work can be formulated in few lines as follows. We formulated the paradigm that two naively unrelated phenomena: local $\mathcal{P}$ violation observed in heavy ion collisions and universal thermal aspects observed in all high energy collisions are in fact both related to quantum anomalies of QCD. The basic idea is that the well established low energy relations representing the quantum anomalies are saturated by extended, thin, coherent, locally low-dimensional sheets of topological charge embedded in 4d space, with opposite sign sheets interleaved. High energy collisions disturb this well-organized structure and lead to emission of physical particles. We argued that a number of long standing puzzles are immediately resolved within this framework. In particular, the thermal spectrum in high energy collisions emerges in spite of the fact that the statistical thermalization could never be reached in those systems. Also: an approximate universality of the temperature with no dependence on energy of colliding particles nor their nature is due to the fact that the emission occurs from the distorted QCD vacuum state rather than from the colliding particles themselves. Finally, as the low-dimensional coherent sheets carry the quantum numbers of the topological charge density, they are responsible for a long range order of the $\mathcal{P}$ odd domains, see section IV B for the details and comparison with available data.

The key point in the analysis of the $\mathcal{P}$ odd correlations is that the distortion of the ground state follows the Casimir type scaling $\sim L^{-p}$ for peripheral collisions for sufficiently large size of the system. This scaling should be contrasted with naive expectation which predicts the exponential type behaviour $\sim \exp \left(-\Lambda_{Q C D} L\right)$. Precisely this Casimir type scaling, in our view, leads to observed, sufficiently strong $\mathcal{P}$ odd correlations (25), while for exponential type behaviour all correlations would be washed out, and numerically much smaller than observed.

The main reason why the Casimir type scaling emerges in a gapped theory such as QCD is the presence of a non-dispersive contribution which is not related to any propagating degrees of freedom, but rather, originated from degenerate topological sectors of the theory. The power like behaviour $\sim L^{-p}$ is, of course, another manifestation of the same long range low-dimensional coherent sheets which are observed on the lattices, and which saturate the low energy relations.

We interpret a relatively large observed intensity of the correlations (25) as a direct manifestation of the Casimir-like scaling and long range order in the QCD vacuum, as these correlations should be strongly suppressed if one takes into account only conventional sources characterized by a finite correlation length $\sim\left(\Lambda_{\mathrm{QCD}}\right)^{-1}$. Future experiments at RHIC and LHC should confirm or rule out this proposal based on the idea of the low-dimensional coherent sheets which are always present in the QCD vacuum as lattice simulations suggest.

It is quite remarkable that some of fundamental properties of QCD, such as Casimir type scaling and long range order, may have profound consequences for cosmology when a relatively small (in QCD units) parameter $L^{-1} \sim\left(10\right.$ fm) ${ }^{-1}$ from application to collisions (25) is replaced by drastically smaller Hubble constant $H \sim 10^{-33} \mathrm{eV}$ in FLRW universe from cosmological application leading to estimate (26). The DE in this framework emerges as mismatch between the energies of a system in a non-trivial FLRW background and Minkowski space-time geometry, similar to the well known Casimir effect. In this framework the DE is entirely rooted into the strongly coupled QCD, without any new fields and/or coupling constants. What is even more remarkable is that some of the most intricate properties of the quantum ground state can be, in principle, experimentally tested in heavy ion collisions at RHIC and LHC, where such unusual environment can be achieved. This idea can be also tested using the lattice Monte Carlo simulations which would allow to study the DE on a computer. 


\section{ACKNOWLEDGEMENTS}

This study had started during the workshop "the first heavy ion collisions at the LHC" at CERN, August 2010, where work [9] had been presented. I had received a numerous number of questions during and after my talk. The basic question was on a relation between formal computations in terms of the acceleration a and description in terms of the gauge fields of underlying gauge theory. This work is the first step to shed some lights on this relation. I am thankful to a number of people including Edward Shuryak, Berndt Mueller, Andrey Leonidov and other participants of the workshop for initiating this study. I am also thankful to James Bjorken for many hours of discussions during his visit to Vancouver. I am also thankful to Ivan Horvath and Folk Bruckmann for correspondence on lattice results and comments on feasibility to measure the Casimir like correction in Monte Carlo simulations. I am also thankful to Dima Kharzeev, Larry McLerran, and other members of Nuclear Physics groups at BNL and Stony Brook U. for useful and stimulating discussions related to the subject of the present work. This research was supported in part by the Natural Sciences and Engineering Research Council of Canada.

\section{Appendix A: On relation of the axion field $a(x)$ with other relevant QCD parameters}

We wish to understand qualitative behaviour of the system when the ground state only slightly deviates from its undisturbed structure as a result of collision. Formally, it corresponds to a study of the system when $a(t, \vec{x}), \eta(t, \vec{x})$ vary very slowly, i.e. we use the derivative expansion. We make an additional simplifying assumption that the system is sufficiently large such that $a(t, \vec{x}), \eta(t, \vec{x})$ do not actually depend on spacial coordinates $\vec{x}$. In this case the problem is reduced to an accounting for the first non-vanishing terms in derivative expansion proportional to $\dot{a}$ and $\dot{\eta}$ which we take as phenomenological parameters for our problem. For this simplified case we want to argue that slow varying axion and dilaton fields $a(x), \eta(x)$ are related to the acceleration "a" $\neq 0$ and some other parameters describing the external sources, see below.

Indeed, any local disturbances resulted from the collision should be described in terms of derivatives $\partial_{\mu} a(x)$ rather than field $a$ itself. This is because $\langle a\rangle=$ const. corresponds to a solution for a minimum of the potential (14) with non-vanishing $\theta$ in entire space, while we are interested in local variations due to the collisions. To simplify things further we consider only time dependent variation $\sim\langle\dot{a}\rangle$ assuming that there is no any coordinate dependence $\vec{x}$ in the system, which corresponds to the collisions of infinitely large ions. For slowly varying axion field in adiabatic approximation one can identify $\langle\dot{a}\rangle=\dot{\theta}_{i n d} / N_{c}$ as it represents a minimum for non vanishing $\theta=$ const. as computed in ref. $[44,45]$.

Furthermore, $\dot{\theta}_{\text {ind }}=\mu_{L}-\mu_{R}$ can be identified with the difference of chemical potentials of the right $\mu_{R}$ and left $\mu_{L}$ handed fermions if they are present in the system, see $[9,16]$ for the details on this identification. In holographic description this parameter is expressed in terms of the boundary condition $\dot{\theta}_{i n d}=A_{0}(z=\infty)$ for the Ramond-Ramond gauge field $A_{0}(z)$ defined in the bulk, see [26] for the details on this identification.

Finally, one can argue that parameter $\dot{\theta}_{\text {ind }}$ should be proportional to the acceleration a when the theory is formulated in geometrical terms. The argument goes like this. We adapt the conjecture formulated in [8] that apparent thermalization observed in high energy collision experiments is a result of tunnelling. In our framework, the quantum tunnelling is happening all the time in vacuum (even before the collision occurs) between the topological sectors, while the observed emission is a result of external impact which causes a non-cancellations between topological extended sheets responsible for the tunnelling, as described in section IV A. Nevertheless, if these tunnelling events can be reformulated eventually in geometrical terms as advocated in [8], the only small parameter which characterizes a small deviation of the system from Minkowski space time is the acceleration a. In conventional QFT description the only parameter which describes the deviation of the system from Minkowski vacuum (14) is $\dot{\theta}_{\text {ind }}$. When both parameters are small, and they both describe the same physics, and they both describe the deviation from perfect Minkowski space time solution (14), they must be proportional to each other, i.e. $\mathbf{a} \sim \dot{\theta}_{\text {ind }}$. Another argument leading to the same conclusion was presented in [9] and was based on description of the $\theta_{\text {ind }}$ state in terms of the Veneziano ghost's fluctuations in accelerating frame which saturate the non-dispersive term in low energy relations as discussed in section III A. The basic idea is that a typical wavelength $\lambda$ of the $\mathcal{P}$ odd fluctuation due to the ghost's fluctuations in the accelerating frame is of order $\lambda \sim 2 \pi / \mathbf{a}$ expressed in terms of acceleration $\mathbf{a}$. A similar scale but expressed in terms of time variating external parameter $\dot{\theta}_{\text {ind }} \sim \omega /(2 \pi)=\lambda^{-1}$ leads to a desired relation $\dot{\theta}_{\text {ind }} \sim \mathbf{a} /(2 \pi)$.

Therefore, in slow varying environment we have the following parameters which all describe the deviation from undisturbed vacuum state (14) and proportional to each other,

$$
\langle\dot{\eta}(x)\rangle \sim\langle\dot{a}(x)\rangle \sim \frac{\dot{\theta}(x)_{\text {ind }}}{N_{c}} \sim \frac{\mathbf{a}(x)}{2 \pi N_{c}} \sim \frac{\left[\mu_{L}(x)-\mu_{R}(x)\right]}{N_{c}} \sim \frac{A_{0}(z=\infty)}{N_{c}} \sim \text { constant } .
$$


Precisely these parameters should play a crucial role in description of the disturbed vacuum state as a result of collision. These parameters in principle accumulate entire information about low energy relations, contact terms, non-dispersive contributions, including the information about the tunnelling events represented by summation over $n$-sectors in path integral and expressed in terms of a "wrong sign" in topological susceptibility (17) in comparison with conventional contribution (18). In case of slowly variational background all these parameters can be approximated by a constant (A1). Therefore, we can use all our previous results formulated in terms of the acceleration [9] if it is sufficiently small, $|\langle\dot{a}\rangle| \sim|\langle\dot{\eta}\rangle| \sim|\mathbf{a}| \ll \Lambda_{Q C D}$. In this approximation one can explicitly see emergence of the thermal spectrum as a result of the Unruh radiation [35] when acceleration a is treated as a constant parameter. We refer to paper [9] where all relevant formulae in the present context with emerging Planck spectrum have been discussed. As we mentioned in the text, the Planck spectrum is a generic feature when the emission is a result of the tunnelling when no information can be transferred. It is very satisfying to see how this spectrum is emergent in the adiabatic limit when the axion field, $\dot{a} \sim \mathbf{a} /\left(2 \pi N_{c}\right)$ varies slowly.

Proportionality of all observables to the acceleration $|\langle\dot{a}\rangle| \sim|\langle\dot{\eta}\rangle| \simeq|\mathbf{a}| \sim T$ is essentially the same effect as Casimir like suppression $L^{-1}$ discussed in the text and Appendix B. Both parameters describe small deviations along the spatial coordinates $L_{x} \sim L_{y} \sim L_{z} \sim L$ or along 4-th coordinate $T^{-1}$, and both parameters $T$ as well as $L^{-1}$ are assumed to be small $\ll \Lambda_{\mathrm{QCD}}$ in derivative expansion. In relativistic system these parameters are essentially the same. In principle, the dynamics of these phenomenological parameters is governed by the dynamics of D2 branes as we discussed in section III. The D2 branes start to fluctuate as a result of the collision, and a small "non-cancellation" effects are effectively described by a small parameter $\mathbf{a}$.

\section{Appendix B: On Casimir like behaviour $1 / L$ in QCD}

There are a number of arguments supporting the Casimir type behaviour $\Delta E \sim L^{-p}$ in QCD demonstrating a huge sensitivity to arbitrary large distances in spite of the presence of a gap in QCD. The difference $\Delta E \equiv\left(E-E_{\text {Mink }}\right)$ here is defined as the difference in energies computed for the system with nontrivial geometry/boundaries and infinite Minkowski space, similar to the well known example of the Casimir effect. This behaviour should be contrasted with conventional computations of the Casimir effect for a massive scalar particle with mass $m$ which leads to the expected exponential scaling $\Delta E \sim \exp (-m L)$, see e.g.[81]. However, as we discussed in the text, along with conventional dispersive contribution in the system, there is also the non-dispersive contribution (19) which emerges as a result of topologically nontrivial sectors in four dimensional QCD. This contact term may lead to a power like corrections $L^{-p}$ rather than exponential like $\exp \left(-\Lambda_{\mathrm{QCD}} L\right)$ because the dispersion relations do not dictate the scaling properties of this term. In different words, a power like behaviour $L^{-p}$ may emerge despite the presence of a mass gap in the system. Such a power-like correction obviously is very unexpected effect which begs for a simple intuitive explanation.

First example is an explicit computation [82] in exactly solvable two- dimensional QED defined in a box size $L$. The model has all elements crucial for present work: non-dispersive contact term which emerges due to the topological sectors of the theory. This model is known to be a theory of a single physical massive field. Still, one can explicitly compute $\Delta E \sim L^{-1}$ which is in drastic contrast with naively expected exponential suppression, $\Delta E \sim e^{-L}$ [82]. One more support in power like behaviour is an explicit computation in a simple case of Rindler space-time in four dimensional QCD $[9,62,83]$ where Casimir like correction have been computed using unphysical Veneziano ghost which effectively describes the dynamics of the topological sectors and the contact term when the background is slightly modified. Thus, power-like behaviour is not a specific feature of two dimensional physics as some people (wrongly) interpret the results [82].

Power like behaviour $\Delta E \sim L^{-p}$ is also supported by recent lattice results [84]. The approach advocated in ref.[84] is based on physical Coulomb gauge when nontrivial topological structure of the gauge fields is represented by the so-called Gribov copies leading to strong infrared singularity. In different words, the same Casimir- like scaling emerges in a different framework where unphysical Veneziano ghost (used in refs. [9, 62, 83]) is not even mentioned.

Similar conclusion also follows from "deformed QCD" model computations where $\Delta E \sim L^{-1}$ correction emerges in a theory where weak coupling regime is enforced by construction and all computations are under complete theoretical control [85]. The very same conclusion also follows from the holographic description of the contact term presented in [55]. The key element for this conclusion follows from the fact that the contact term in holographic description is determined by massless Ramond-Ramond (RR) gauge field defined in the bulk of 5-dimensional space. Therefore, it is quite natural to expect that massless R-R field in holographic description leads to power like corrections when the background is slight modified.

As this effect plays a crucial role in the applications considered in section IV, we want to present here few other systems where a similar phenomena occurs, and where it has precisely the same nature. Furthermore, in these systems a similar problem can be exactly solved (in drastic contrast with strongly coupled 4d QCD). Most importantly, an analogous effect in these systems has been experimentally observed. 
We start from the well known Aharonov -Casher effect as formulated in [86]. The relevant part of this work can be stated as follows. If one inserts an external charge into superconductor when the electric field is exponentially suppressed $\sim \exp (-r / \lambda)$ with $\lambda$ being the penetration depth, a neutral magnetic fluxon will be still sensitive to an inserted external charge at arbitrary large distance. The effect is pure topological and non-local in nature. The crucial element why this phenomenon occurs in spite of the fact that the system is gapped is the presence of different topological states in the system. We do not have a luxury to solve a similar problem in strongly coupled four dimensional QCD analytically. However, one can argue that the role of "modular operator" of [86] (which is the key element in demonstration of long range order) is played by large gauge transformation operator $\mathcal{T}$ in QCD which also commutes with the hamiltonian $[\mathcal{T}, H]=0$, such that our system must be transparent to topologically nontrivial pure gauge configurations, similar to transparency of the superconductor to the "modular electric field", see [55] for the details. Such a behaviour of our system can be thought as a non-local topological effect similar to the non-local Aharonov -Casher effect. The last word whether this analogy can be extended to the strongly coupled four dimensional QCD remains, of course, the prerogative of the direct lattice computations. We should mention that there are few other systems, such as topological insulators, where a topological long range order emerges in spite of the presence of a gap in the system. We refer to ref [55] for relevant references and details.

[1] S. A. Voloshin, Phys. Rev. C 70, 057901 (2004) [arXiv:hep-ph/0406311].

I. V. Selyuzhenkov [STAR Collaboration], Rom. Rep. Phys. 58, 049 (2006) [arXiv:nucl-ex/0510069].

S. A. Voloshin [STAR Collaboration], arXiv:0806.0029 [nucl-ex], arXiv:1006.1020 [nucl-th].

B. I. Abelev et al. [STAR Collaboration], Phys. Rev. Lett. 103, 251601 (2009) [arXiv:0909.1739 [nucl-ex]].

N. N. Ajitanand, S. Esumi, R. A. Lacey [PHENIX Collaboration], in: Proc. of the RBRC Workshops, vol. 96, 2010: P- and $\mathrm{CP}$-odd effects in hot and dense matter.

B. Mohanty et al [STAR Collaboration], [arXiv:1106.5902 [nucl-ex]].

[2] B. I. Abelev et al. [STAR Collaboration], Phys. Rev. C 81, 054908 (2010) [arXiv:0909.1717 [nucl-ex]].

[3] P. Christakoglou for the ALICE Collaboration, J. Phys. G G 38, 124165 (2011) [arXiv:1106.2826 [nucl-ex]].

[4] I. Selyuzhenkov [ALICE Collaboration], arXiv:1111.1875 [nucl-ex];

I. Selyuzhenkov [ALICE Collaboration], arXiv:1203.5230 [nucl-ex].

[5] D. Kharzeev, Phys. Lett. B 633, 260 (2006) [arXiv:hep-ph/0406125].

[6] D. Kharzeev and A. Zhitnitsky, Nucl. Phys. A 797, 67 (2007) [arXiv:0706.1026 [hep-ph]].

[7] R. Hagedorn, Nuovo Cim. Suppl. 3 (1965) 147; Nuovo Cim. A 56 (1968) 1027.

[8] P. Castorina, D. Kharzeev and H. Satz, Eur. Phys. J. C 52, 187 (2007) [arXiv:0704.1426 [hep-ph]].

[9] A. R. Zhitnitsky, Nucl. Phys. A853, 135-163 (2011). [arXiv:1008.3598 [nucl-th]].

[10] D. Kharzeev, R. D. Pisarski and M. H. G. Tytgat, Phys. Rev. Lett. 81, 512 (1998) [arXiv:hep-ph/9804221].

[11] D. Kharzeev and R. D. Pisarski, Phys. Rev. D 61, 111901 (2000) [arXiv:hep-ph/9906401].

[12] K. Buckley, T. Fugleberg and A. Zhitnitsky, Phys. Rev. Lett. 84, 4814 (2000) [arXiv:hep-ph/9910229].

[13] K. Buckley, T. Fugleberg and A. Zhitnitsky, Phys. Rev. C 63, 034602 (2001) [arXiv:hep-ph/0006057].

[14] D. E. Kharzeev, L. D. McLerran and H. J. Warringa, Nucl. Phys. A 803, 227 (2008) [arXiv:0711.0950 [hep-ph]].

[15] K. Fukushima, D. E. Kharzeev and H. J. Warringa, Phys. Rev. D 78, 074033 (2008) [arXiv:0808.3382 [hep-ph]].

[16] D. E. Kharzeev, Annals Phys. 325, 205 (2010) [arXiv:0911.3715 [hep-ph]].

[17] P. V. Buividovich, M. N. Chernodub, E. V. Luschevskaya and M. I. Polikarpov, Phys. Rev. D 80, 054503 (2009) [arXiv:0907.0494 [hep-lat]].

[18] M. Abramczyk, T. Blum, G. Petropoulos and R. Zhou, PoS LAT2009, 181, (2009) arXiv:0911.1348 [hep-lat].

[19] A. Vilenkin, Phys. Rev. D 20, 1807 (1979); Phys. Rev. D 22, 3067 (1980); Phys. Rev. D 22, 3080 (1980).

[20] A. Yu. Alekseev, V. V. Cheianov and J. Fröhlich, Phys. Rev. Lett. 81, 3503 (1998).

[21] J. Charbonneau and A. Zhitnitsky, Phys. Rev. C 76, 015801 (2007) [arXiv:astro-ph/0701308].

[22] J. Charbonneau and A. Zhitnitsky, JCAP 1008, 010 (2010) [arXiv:0903.4450 [astro-ph.HE]].

[23] D. T. Son and A. R. Zhitnitsky, Phys. Rev. D 70, 074018 (2004) [arXiv:hep-ph/0405216].

[24] M. A. Metlitski and A. R. Zhitnitsky, Phys. Rev. D 72, 045011 (2005) [arXiv:hep-ph/0505072].

[25] G. Lifschytz and M. Lippert, Phys. Rev. D 80 (2009) 066005, [0904.4772];

H. U. Yee, JHEP 0911, 085 (2009) [arXiv:0908.4189 [hep-th]];

A. Rebhan, A. Schmitt and S. A. Stricker, JHEP 1001, 026 (2010) [arXiv:0909.4782 [hep-th]];

A. Gorsky, P. N. Kopnin and A. V. Zayakin, Phys. Rev. D 83, 014023 (2011) [arXiv:1003.2293 [hep-ph]];

A. Gynther, K. Landsteiner, F. Pena-Benitez and A. Rebhan, JHEP 1102, 110 (2011) [arXiv:1005.2587 [hep-th]].

V. A. Rubakov, arXiv:1005.1888 [hep-ph].

[26] L. Brits, J. Charbonneau, Phys. Rev. D 83, 126013 (2011) [arXiv:1009.4230 [hep-th]].

[27] D. E. Kharzeev, [arXiv:1107.4004 [hep-ph]].

[28] A. Bzdak, V. Koch and J. Liao, Phys. Rev. C 81, 031901 (2010) [arXiv:0912.5050 [nucl-th]].

[29] J. Liao, V. Koch and A. Bzdak, Phys. Rev. C 82, 054902 (2010) [arXiv:1005.5380 [nucl-th]].

[30] A. Bzdak, V. Koch and J. Liao, Phys. Rev. C 83, 014905 (2011) [arXiv:1008.4919 [nucl-th]]. 
[31] S. Schlichting and S. Pratt, Phys. Rev. C 83, 014913 (2011) [arXiv:1009.4283 [nucl-th]].

[32] S. Pratt, S. Schlichting and S. Gavin, Phys. Rev. C 84, 024909 (2011) [arXiv:1011.6053 [nucl-th]].

[33] M. Asakawa, A. Majumder and B. Muller, Phys. Rev. C 81, 064912 (2010) [arXiv:1003.2436 [hep-ph]].

[34] A. Salam and J. Strathdee, Phys. Lett. B 66 (1977) 143;

S. Barshay and W. Troost, Phys. Lett. B 73 (1978) 437;

A. Hosoya, Progr. Theoret. Phys. 61 (1979) 280;

M. Horibe, Progr. Theoret. Phys. 61 (1979) 661;

[35] N. D. Birrell and P. C. W. Davies, Quantum Fields In Curved Space, Cambridge Univ. Pr. , 1982.

[36] D. Kharzeev and K. Tuchin, Nucl. Phys. A 753, 316 (2005) [arXiv:hep-ph/0501234].

D. Kharzeev, Nucl. Phys. A 774, 315 (2006) [arXiv:hep-ph/0511354].

D. Kharzeev, E. Levin and K. Tuchin, Phys. Rev. C 75, 044903 (2007) [arXiv:hep-ph/0602063].

D. Kharzeev, Eur. Phys. J. A 29, 83 (2006).

H. Satz, Eur. Phys. J. ST 155, 167 (2008).

F. Becattini, P. Castorina, J. Manninen and H. Satz, Eur. Phys. J. C 56, 493 (2008) [arXiv:0805.0964 [hep-ph]].

P. Castorina, D. Grumiller and A. Iorio, Phys. Rev. D 77, 124034 (2008) [arXiv:0802.2286 [hep-th]].

P. Steinberg, Acta Phys. Hung. A 24, 51 (2005) [arXiv:nucl-ex/0405022].

N. Evans and A. Tedder, Phys. Rev. Lett. 100, 162003 (2008) [arXiv:0711.0300 [hep-ph]].

Y. Hatta and T. Matsuo, Phys. Rev. Lett. 102, 062001 (2009) [arXiv:0807.0098 [hep-ph]].

[37] V. A. Novikov, M. A. Shifman, A.I. Vainshtein and V. I. Zakharov, Nucl. Phys. B 191, 301 (1981)

[38] G. Veneziano, Nucl. Phys. B 159, 213 (1979).

[39] P. Di Vecchia and G. Veneziano, Nucl. Phys. B 171, 253 (1980).

[40] E. Witten, Nucl. Phys. B 156, 269 (1979).

[41] C. Rosenzweig, J. Schechter, C. G. Trahern, Phys. Rev. D21, 3388 (1980).

[42] P. Nath, R. L. Arnowitt, Phys. Rev. D23, 473 (1981).

[43] K. Kawarabayashi, N. Ohta, Nucl. Phys. B175, 477 (1980).

[44] I. E. Halperin, A. Zhitnitsky, Phys. Rev. D58, 054016 (1998). [hep-ph/9711398].

[45] I. E. Halperin, A. Zhitnitsky, Phys. Rev. Lett. 81, 4071-4074 (1998). [hep-ph/9803301].

[46] E. Thomas and A. R. Zhitnitsky, Phys. Rev. D 85, 044039 (2012) [arXiv:1109.2608 [hep-th]].

[47] M. Ünsal and L. G. Yaffe, Phys. Rev. D 78, 065035 (2008). [arXiv:0803.0344 [hep-th]].

[48] A. R. Zhitnitsky, Phys. Rev. D84, 124008 (2011). [arXiv:1105.6088 [hep-th]].

[49] A. A. Migdal and M. A. Shifman, Phys. Lett. B114, 445 (1982).

[50] D. Kharzeev, E. Levin and K. Tuchin, Phys. Lett. B 547, 21 (2002) [arXiv:hep-ph/0204274].

[51] D. Kharzeev, E. Levin and K. Tuchin, Phys. Rev. D 70, 054005 (2004) [arXiv:hep-ph/0403152].

[52] D. Kharzeev, E. Levin and K. Tuchin, JHEP 0906, 055 (2009) [arXiv:0809.3794 [hep-ph]].

[53] M. Srednicki (2002), arXiv:hep-th/0210172;

K. van Bibber, L. J. Rosenberg, Phys. Today 59N8, 30-35 (2006);

S. J. Asztalos, L. J. Rosenberg, K. van Bibber, P. Sikivie, K. Zioutas, Ann. Rev. Nucl. Part. Sci. 56, 293-326 (2006).

[54] E. Witten, Phys. Rev. Lett. 812862 (1998) [arXiv:hep-th/9807109].

[55] A. R. Zhitnitsky, "Contact Term, its Holographic Description in QCD and Dark Energy," arXiv:1112.3365 [hep-ph].

[56] I. Horvath, A. Alexandru, J. B. Zhang, Y. Chen, S. J. Dong, T. Draper, K. F. Liu, N. Mathur et al., Phys. Lett. B617, 49-59 (2005). [hep-lat/0504005].

[57] E. -M. Ilgenfritz, K. Koller, Y. Koma, G. Schierholz, T. Streuer and V. Weinberg, Phys. Rev. D 76, 034506 (2007) [arXiv:0705.0018 [hep-lat]].

[58] E. -M. Ilgenfritz, D. Leinweber, P. Moran, K. Koller, G. Schierholz and V. Weinberg, Phys. Rev. D 77, 074502 (2008) [Erratum-ibid. D 77, 099902 (2008)] [arXiv:0801.1725 [hep-lat]].

[59] F. Bruckmann, N. Cundy, F. Gruber, T. Lippert and A. Schafer, Phys. Lett. B707, 278 (2012). arXiv:1107.0897 [hep-lat].

[60] C. Bernard et al, PoS (LATTICE 2007) 310 [arXiv:0710.3124 hep-lat].

[61] T. Schafer and E. V. Shuryak, Rev. Mod. Phys. 70, 323 (1998) [hep-ph/9610451].

[62] A. R. Zhitnitsky, Phys. Rev. D 82, 103520 (2010), arXiv:1004.2040 [gr-qc].

[63] I. Horvath, S. J. Dong, T. Draper, F. X. Lee, K. F. Liu, N. Mathur, H. B. Thacker, J. B. Zhang, Phys. Rev. D68, 114505 (2003). [hep-lat/0302009].

[64] I. Horvath, A. Alexandru, J. B. Zhang, Y. Chen, S. J. Dong, T. Draper, F. X. Lee, K. F. Liu et al., Phys. Lett. B612, 21-28 (2005). [hep-lat/0501025].

[65] A. Alexandru, I. Horvath and J. -b. Zhang, Phys. Rev. D 72, 034506 (2005) [hep-lat/0506018].

[66] S. Ahmad, J. T. Lenaghan, H. B. Thacker, Phys. Rev. D72, 114511 (2005). [hep-lat/0509066].

[67] A. Gorsky, V. Zakharov, Phys. Rev. D77, 045017 (2008). [arXiv:0707.1284 [hep-th]].

[68] A. S. Gorsky, V. I. Zakharov, A. R. Zhitnitsky, Phys. Rev. D79, 106003 (2009). [arXiv:0902.1842 [hep-ph]].

[69] H. Verschelde, V. I. Zakharov, [arXiv:1107.1393 [hep-th]].

[70] E. Witten, "Anti-de Sitter space, thermal phase transition, and confinement in gauge theories," Adv. Theor. Math. Phys. 2, 505 (1998) [arXiv:hep-th/9803131].

[71] A. M. Steinberg, P. G. Kwiat and R. Y. Chiao, Phys. Rev. Lett. 71, 708 (1993).

[72] R. Y. Chiao, "Tunneling times and superluminality: A Tutorial," arXiv:quant-ph/9811019.

[73] K. Fukushima, J. Phys. G G 39, 013101 (2012) [arXiv:1108.2939 [hep-ph]]. 
[74] D. M. Ostrovsky, G. W. Carter and E. V. Shuryak, Phys. Rev. D 66, 036004 (2002) [hep-ph/0204224].

[75] E. Shuryak, Nucl. Phys. A 715, 289 (2003) [hep-ph/0205031].

[76] G. Basar, D. E. Kharzeev, H. -U. Yee and I. Zahed, arXiv:1202.0831 [hep-th].

[77] S. W. Hawking and G. T. Horowitz, Class. Quant. Grav. 13, 1487 (1996) [arXiv:gr-qc/9501014].

[78] F. Belgiorno and S. Liberati, Gen. Rel. Grav. 29, 1181 (1997) [arXiv:gr-qc/9612024].

[79] Y. B. Zeldovich, JETP Lett. 6, 316 (1967) [Pisma Zh. Eksp. Teor. Fiz. 6, 883 (1967)].

[80] F. R. Urban and A. R. Zhitnitsky, Nucl. Phys. B 835, 135 (2010) [arXiv:0909.2684 [astro-ph.CO]]; Phys. Lett. B 688, 9 (2010) [arXiv:0906.2162 [gr-qc]].

[81] G. Plunien, B. Muller and W. Greiner Phys. Rep. 134, 87 (1986).

[82] F. R. Urban, A. R. Zhitnitsky, Phys. Rev. D80, 063001 (2009). [arXiv:0906.2165 [hep-th]].

[83] N. Ohta, Phys. Lett. B 695, 41 (2011) [arXiv:1010.1339 [astro-ph.CO]].

[84] B. Holdom, Phys. Lett. B697, 351-356 (2011). [arXiv:1012.0551 [hep-ph]].

[85] E. Thomas and A. R. Zhitnitsky, arXiv:1203.6073 [hep-ph].

[86] B. Reznik, Y. Aharonov, Phys. Rev. D40, 4178-4183 (1989). 